\title{
Therapeutic Options for Patients with Refractory Status Epilepticus in Palliative Settings or with a Limitation of Life-Sustaining Therapies: A Systematic Review
}

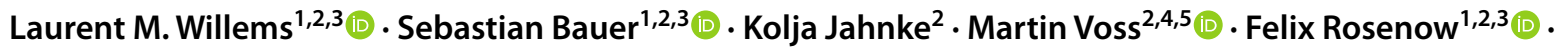 \\ Adam Strzelczyk $\mathbf{k}^{1,2,3,6}$
}

Published online: 23 July 2020

(c) The Author(s) 2020, Corrected publication 2021

\begin{abstract}
Background Refractory status epilepticus (RSE) represents a serious medical condition requiring early and targeted therapy. Given the increasing number of elderly or multimorbid patients with a limitation of life-sustaining therapy (LOT) or within a palliative care setting (PCS), guidelines-oriented therapy escalation options for RSE have to be omitted frequently.

Objectives This systematic review sought to summarize the evidence for fourth-line antiseizure drugs (ASDs) and other minimally or non-invasive therapeutic options beyond guideline recommendations in patients with RSE to elaborate on possible treatment options for patients undergoing LOT or in a PCS.

Methods A systematic review of the literature in accordance with the Preferred Reporting Items for Systematic Reviews and Meta-Analyses (PRISMA) guidelines, focusing on fourth-line ASDs or other minimally or non-invasive therapeutic options was performed in February and June 2020 using the MEDLINE, EMBASE and Cochrane databases. The search terminology was constructed using the name of the specific ASD or therapy option and the term 'status epilepticus' with the use of Boolean operators, e.g. "(brivaracetam) AND (status epilepticus)". The respective Medical Subject Headings (MeSH) and Emtree terms were used, if available.

Results There is currently no level 1, grade A evidence for the use of ASDs in RSE. The best evidence was found for the use of lacosamide and topiramate (level 3, grade C), followed by brivaracetam, perampanel (each level 4, grade D) and stiripentol, oxcarbazepine and zonisamide (each level 5, grade D). Regarding non-medicinal options, there is little evidence for the use of the ketogenic diet (level 4, grade D) and magnesium sulfate (level 5, grade D) in RSE. The broad use of immunomodulatory or immunosuppressive treatment options in the absence of a presumed autoimmune etiology cannot be recommended; however, if an autoimmune etiology is assumed, steroid pulse, intravenous immunoglobulins and plasma exchange/plasmapheresis should be considered (level 4, grade D). Even if several studies suggested that the use of neurosteroids (level 5, grade D) is beneficial in RSE, the current data situation indicates that there is formal evidence against it.

Conclusions RSE in patients undergoing LOT or in a PCS represents a challenge for modern clinicians and epileptologists. The evidence for the use of ASDs in RSE beyond that in current guidelines is low, but several effective and well-tolerated options are available that should be considered in this patient population. More so than in any other population, advance care planning, advance directives, and medical ethical aspects have to be considered carefully before and during therapy.
\end{abstract}

Laurent M. Willems

laurent.willems@kgu.de

Extended author information available on the last page of the article 


\section{Key Points}

There is sustainable evidence for the efficacy of fourthline antiseizure drugs (ASDs) in status epilepticus (SE) and refractory SE (RSE).

Lacosamide, topiramate, perampanel, brivaracetam, and stiripentol should be considered in patients with RSE in limited therapy settings.

Besides ASDs, the ketogenic diet can be a therapy option that must be individually evaluated for every case.

There is minor evidence for the use of magnesium sulfate in non-eclamptic SE as an additional therapeutic approach.

A central part of any medical therapeutic decision in RSE is the reflection of medical ethical aspects in the context of the patient's will.

\section{Introduction}

Status epilepticus (SE) is a frequent and potentially lifethreatening medical condition that must be treated immediately to prevent further harm from befalling the affected patient. SE can not only manifest in patients with known epilepsy but can also have many other triggers, such as infection, brain damage, electrolyte imbalance, and autoimmune or paraneoplastic antibodies [1, 2]. According to German and other national and international clinical practice guidelines, benzodiazepines (BZDs) represent the first-line therapy in the treatment of SE. The International League Against Epilepsy defines all tonic-clonic seizures lasting for more than $5 \mathrm{~min}$, as well as focal seizures with impaired consciousness or absence seizures lasting for more than $10 \mathrm{~min}$, as SE [3]. An SE that lasts for more than 10 min despite adequately administered therapy with BZDs should be treated with intravenous antiseizure drugs (ASDs) such as levetiracetam (LEV), valproic acid (VPA), or (fos-)phenytoin (PHT) [4]. In the recently published ESETT trial, all three drugs showed similar efficacy profiles, at 47\% (LEV), $45 \%$ (fosPHT), and 46\% (VPA), in terminating an established SE, while having similar incidence rates of adverse events (AEs) [4]. If the combination of one BZD and one ASD fails, then criteria for a refractory SE (RSE) are met and further treatment in the intensive care unit (ICU), as well as the consequent use of anesthetic drugs together with the need for mechanical ventilation, are recommended for generalized tonic-clonic SE [5-8]. Failure to control SE with anesthetic drugs defines a super-refractory SE (SRSE) that is associated with increased mortality, decreased quality of life, and poor chance of response to further therapeutics [2, 9-14]. Due to demographic changes such as an increasing number of elderly and multimorbid patients $[15,16]$, as well as the growing importance of self-determination for, in particular, severely ill patients within a palliative care setting (PCS) or with another limitation of life-sustaining therapy (LOT) [17], most of the recommended therapeutic options for RSE and SRSE fail due to invasiveness or the requirement of an ICU/IMC stay, invasive monitoring or mechanical ventilation $[18,19]$. Moreover, based on the increasing implementation of advanced care planning (ACP), many patients have decided already in times of good health conditions or at the onset of a chronic or potentially life-threatening disease to waive specific medical treatment options [20]. However, many patients and their relatives or caregivers insist on a therapeutic attempt in spite of the given LOT or PCS, as SE per se does not always result in an additional permanent disability [21]. Moreover, many patients with acute or preexisting relevant cardiopulmonary or hepatorenal conditions cannot be treated with several second- or third-line ASDs due to their high interaction potential (i.e. with phenobarbital [PB], PHT, or VPA), proarrhythmic effects (e.g. PHT), or contraindications to their use in combination with other drugs (e.g. enhanced VPA metabolism due to carbapenem antibiotics) [5, 19, 22].

This systematic review sought to summarize the evidence for fourth-line ASDs and other minimally or non-invasive therapeutic options beyond guideline recommendations in patients with RSE to elaborate on possible treatment options for patients undergoing LOT or in a PCS.

\section{Methods}

\subsection{Characteristics of the Review}

To evaluate the possible therapeutic options for patients undergoing ACP or LOT, or in a PCS, beyond the guideline recommendations, a systematic review of the literature was performed in February 2020 using the MEDLINE, EMBASE, and Cochrane databases, using the PubMed gateway, focusing on minimally or non-invasive treatment options that do not require invasive monitoring or admission to the ICU. Based on reviewers' recommendations, a second literature study was performed in June 2020 focusing on immunomodulatory and immunosuppressive treatment options. Studies with patients of all age ranges were included. The Preferred Reporting Items for Systematic Reviews and Meta-Analyses (PRISMA) guidelines were closely followed; the PRISMA checklist for this review is available on request $[23,24]$. The search terminology was 
constructed using the name of the specific ASD or therapy option and the term 'status epilepticus' with the use of Boolean operators, e.g. "(brivaracetam) AND (status epilepticus)". The respective Medical Subject Headings (MeSH) and Emtree terms were used, if available. The protocol for this systematic review has not been previously registered on PROSPERO [25]. As other minimally or non-invasive therapeutic options, the intravenous application of magnesium sulfate $\left(\mathrm{MgSO}_{4}\right)$, neurosteroids (e.g. allopregnanolone), and the ketogenic diet (KD), as well as steroid pulse (SP), intravenous immunoglobulins (IVIGs) and plasma exchange/ plasmapheresis (PLEX), were considered based on recent publications $[1,2]$. No further filter was applied to avoid the falsification of the results, and two reviewers (LMW, AS) screened the identified articles for eligibility. Publications were defined as 'relevant' if the following criteria were met: (1) original publication; (2) human study; (3) at least one enrolled patient; and (4) sufficient data to enable a comparison with other publications. Reasons to exclude publications from this review included the unavailability of a version in English, French, German, Italian, or Spanish. Available reviews with a total of over 300 enrolled subjects for the use of a single ASD or other therapeutic options were used to cross-check the selected publications [26-41]. Processed data (e.g. number of subjects, age range, study design, characterization of SE, severity of SE, loading dose, maintenance dose, responder rate, rate of AEs in studies on ASDs) of the individual studies were manually transferred into a previously conceived assessment form. In addition, other aspects such as nutrition regimen, number of patients achieving ketosis (of those on $\mathrm{KD}$ ), treatment days, relapse of seizures after the discontinuation of therapy $\left(\mathrm{MgSO}_{4}\right)$, treatment days, and titration and maintenance (if taking neurosteroids) doses were accessed. Regarding immunomodulatory and immunosuppressive therapies, treatment options and the specific response was recorded. Moreover, individual aspects of particular interest, and the strengths and weaknesses of each study, were assessed. Due to the use of processed data, cross-checking for double entries of single individuals was impossible and no additional post hoc subgroup analysis was performed. The risk of a specific bias was assessed for each publication; however, due to the low number of available publications with predominantly small numbers of enrolled patients, no studies were excluded, and major biases were discussed, except one study with previously proven fundamental statistical errors. The responder rate was defined as the primary outcome, while safety and tolerability (AEs) were defined as secondary outcomes for this study.

\subsection{Level of Evidence}

The well-established and internationally accepted Oxford classification scheme from the Oxford Centre for EvidenceBased Medicine (CEBM; March 2009) [42] was used to graduate the existing evidence based on the available quality and design of the published studies and reports in the following order.

(1) Randomized controlled trials (RCT) or meta-analyses of RCTs with homogenous results.

(2) Poorly designed RCTs, prospective cohort studies, or meta-analyses of level 2 studies.

(3) Retrospective cohort studies, case-control studies, or meta-analyses of level 3 studies.

(4) Case series.

(5) Case reports, expert opinions, or personal observations.

Based on the available amount, quality, and consistency of the available publications, the following different grades of recommendation can be concluded.

(A) Consistent level 1 studies.

(B) Consistent level 2 or 3 studies or extrapolations from level 1 studies.

(C) Level 4 studies or extrapolations from level 2 or 3 studies.

(D) Level 5 evidence or troublingly inconsistent or inconclusive studies of any level.

In addition to the Oxford classification scheme, which focuses on the existing evidence, the Grading Recommendations Assessment, Development, and Evaluation (GRADE) scale was adopted to access the strength of recommendations [43]. The GRADE significance is subdivided into the following four quality levels.

(I) 'High', wherein we are very confident that the true effect lies close to that of the estimate of the effect.

(II) 'Moderate', wherein we are moderately confident in the effect estimate; the true effect is likely to be close to the estimate of the effect but there is a possibility that it is substantially different.

(III) 'Low', wherein our confidence in the effect estimate is limited; the true effect may be substantially different from the estimate of the effect.

(IV) 'Very low', wherein we have very little confidence in the effect estimate; the true effect is likely to be substantially different from the estimate of the effect. 
The displayed level of evidence and grade of recommendation for each ASD or therapeutic option represents the consensus of all authors of this publication.

\subsection{Definitions and Terminology}

Definitions and terminology for seizures, epilepsy, and SE used for this review follow the international recommendations of the International League against Epilepsy [3, 44, 45]. Due to the method-inherent character of this systematic review, no reappraisal of the reported responder or $\mathrm{AE}$ rates was performed, which means that all patients with a reported response or $\mathrm{AE}$ were counted without any further subcategorization. If AEs were not explicitly named or their absence was reported, AEs were classified as not available. Definitions and terms in relation to therapeutic restrictions, palliation, and end-of life care follow the current doctrines $[20,46]$ (please refer to the Infobox for more information).

\section{Infobox}

Advance Care Planning (ACP): ACP describes the process that enables individuals to plan their future healthcare by providing directions to caretakers, relatives, and healthcare professionals when the affected person is not in a position to make or communicate their own healthcare choices [20]

Limitation of Life-Sustaining Therapy (LOT): LOT is a therapy setting derived from decisions by individual ACPs that restrict the adoption of specific medical options in the case of deterioration in health status. Typical examples of a LOT are the renunciation of treatment in the intensive care unit, mechanical ventilation or intubation, organ replacement procedures, or resuscitation in the case of cardiac arrest [20]

Palliative Care Setting (PCS): PCS is a defined as a limited treatment regimen that focuses on treatment strategies for improving the quality of life of patients and their caregivers in patients with life-threatening illnesses and unfavorable prognoses through the prevention and relief of suffering [46]

\subsection{Data Analysis and Reporting}

All numbers with decimals were rounded to the nearest full number except those of dose recommendations in pediatric patients. No further data analysis or processing was performed for this systematic review.

\section{Results}

\subsection{General Aspects}

A total of 1481 publications were screened, resulting in the identification of 191 (13\%) publications that were relevant to the topic of this systematic review. For more details on the PRISMA diagram, please refer to Fig. 1. Of these 191 publications, 62 (32\%) focused on ASDs and 129 (68\%) focused on other therapeutic options. Among fourth-line ASDs, the highest number of relevant publications was available for lacosamide (LCM; $n=27,44 \%$ ), followed by topiramate (TPM; $n=15,24 \%$ ), perampanel (PER; $n=9,15 \%$ ), brivaracetam (BRV; $n=5,8 \%$ ), stiripentol (STP; $n=3,5 \%)$ and lamotrigine (LTG), oxcarbazepine (OXC) or zonisamide (ZNS; $n=1$ each, 2\%). Regarding other therapeutic options, most relevant publications reported on immunomodulatory and immunosuppressive therapies $(n=72,65 \%)$, followed by the $\mathrm{KD}(n=30,23 \%), \mathrm{MgSO}_{4}(n=23,18 \%)$ and neurosteroids $(n=4,3 \%)$.

\subsection{Antiseizure Drugs (ASDs)}

BRV is a novel third-generation ASD available in tablet, oral, and intravenous solution forms and is approved for monotherapy (US FDA) or add-on therapy (European Medicines Agency) in focal epilepsy (FE). BRV is a derivate of LEV, acting mainly as a ligand at the synaptic vesicle glycoprotein $2 \mathrm{~A}$ (SV2A). In comparison with LEV, BRV has a tenfold increased affinity to its molecular target and boasts a lower potential for inducing psychobehavioral adverse effects [37, 47, 48]. BRV is not approved for use in SE; the evidence for its use is limited to five retrospective case series and reports offering a total of 77 adult cases with responder rates for convulsive SE (CSE) and non-convulsive SE (NCSE) of between 27 and 54\%, as well as an acceptable tolerability profile [49-53]. The largest available retrospective multicenter cohort included 43 adult patients and reported a responder rate of $54 \%$ as well as good tolerability, with AEs observed in 14\% of the enrolled subjects [50]. Two studies provided a subgroup analysis of a connection between responders and dosage showing a significant correlation of a response with a loading dose of $1.82-2.0 \mathrm{mg} /$ $\mathrm{kg}$ body weight or greater $[50,53]$. According to the current state of knowledge, a loading dose of 50-400 mg, considering the given minimum dose of approximately $2 \mathrm{mg} / \mathrm{kg}$ body weight and a maintenance dose of $100-400 \mathrm{mg} / \mathrm{day}$, has been shown to be well tolerated and effective (Table $1 b$ ).

Carbamazepine (CBZ) is a second-generation ASD available in tablet and oral solution forms only, unfolding its anticonvulsive effect via voltage-gated sodium $\left(\mathrm{Na}^{+}\right)$channels and being approved for both monotherapy and add-on 


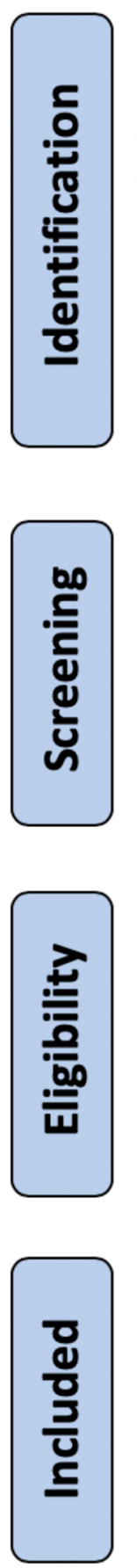

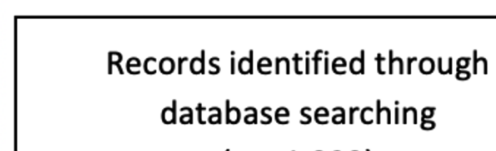

$(n=1,392)$

\section{Additional records identified}

through other sources

$(n=89)$
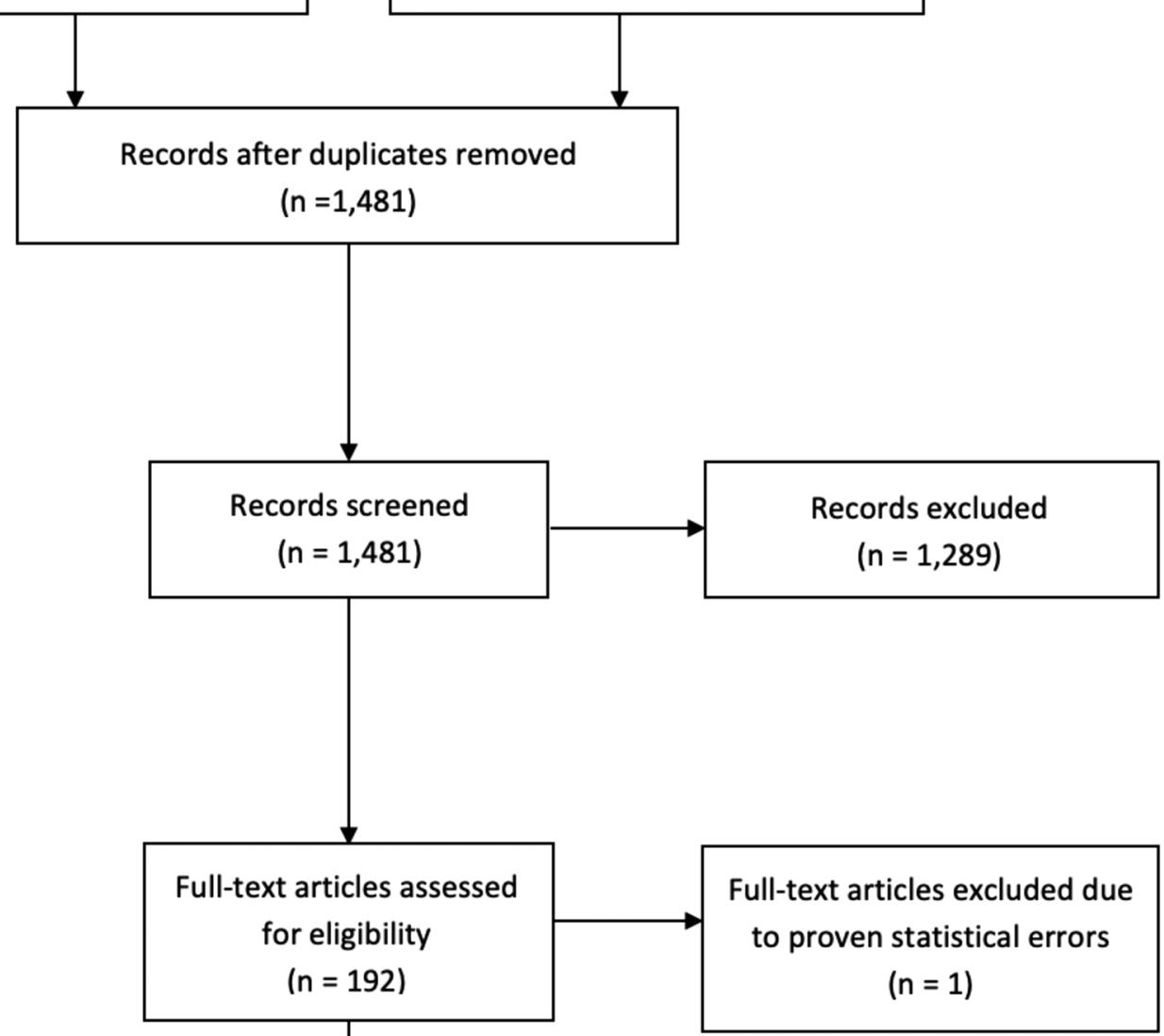

Studies included in

qualitative synthesis

$(n=191)$

Fig. 1 PRISMA flow diagram. PRISMA Preferred Reporting Items for Systematic Reviews and Meta-Analyses

therapy in FE [54]. There is currently no evidence supporting the efficacy, safety, or tolerability of CBZ in SE.

Eslicarbazepine (ESL) is a third-generation ASD approved for monotherapy and add-on therapy for FE, and is available in an oral formulation with the same mechanism of action as that of OXC, i.e. the modulation of voltage-gated $\mathrm{Na}^{+}$channels [54]. There is currently no evidence supporting the efficacy, safety, or tolerability of ESL in SE.
LCM is a third-generation ASD available in tablet, oral, and intravenous forms and is approved for both monotherapy and add-on therapy in FE, which unfolds its effect by antagonism at voltage-gated $\mathrm{Na}^{+}$channels. LCM is currently not approved for use in SE. There are 20 retrospective case reports or series, as well as seven prospective studies, for a total of 862 pediatric and adult patients with CSE and NCSE, showing a responder rate ranging from 0 to $100 \%$. 
Table 1 Current evidence for the use of second- and third-line ASDs in refractory and super-refractory status epilepticus

\begin{tabular}{|c|c|c|c|c|c|c|c|c|c|}
\hline & \multicolumn{3}{|c|}{ Study design } & \multicolumn{2}{|c|}{ Status epilepticus } & \multicolumn{2}{|l|}{ Therapy regimen } & \multicolumn{2}{|l|}{ Outcome } \\
\hline & \multirow[t]{2}{*}{ Layout } & \multicolumn{2}{|c|}{ Study population } & \multirow[t]{2}{*}{ Type } & \multirow[t]{2}{*}{ Severity } & \multirow[t]{2}{*}{ Loading dose (mg) } & \multirow{2}{*}{$\begin{array}{l}\text { Maintenance } \\
\text { dose (mg/ } \\
\text { day) }\end{array}$} & \multirow[t]{2}{*}{ Response (\%) } & \multirow[t]{2}{*}{ AEs $(\%)$} \\
\hline & & $n$ & Age (years) & & & & & & \\
\hline \multicolumn{10}{|c|}{ (a) Lacosamide (LCM) } \\
\hline $\begin{array}{l}\text { Kellinghaus et al. } \\
\text { (2011) [55] }\end{array}$ & $\mathrm{rs}, \mathrm{mc}$ & 39 & $18-90$ & CSE & SE, RSE & $200-400$ & - & 44 & 77 \\
\hline $\begin{array}{l}\text { Albers et al. (2011) } \\
\text { [56] }\end{array}$ & rs, sc & 7 & $33-83$ & CSE & SE, RSE & 400 & 400 & 100 & - \\
\hline $\begin{array}{l}\text { Goodwin et al. } \\
\text { (2011) [57] }\end{array}$ & rs, sc & 9 & $47-89$ & CSE, NCSE & RSE & 200 & 400 & 0 & 22 \\
\hline $\begin{array}{l}\text { Höfler et al. (2011) } \\
\text { [58] }\end{array}$ & $\mathrm{rs}, \mathrm{mc}$ & 31 & $22-95$ & CSE, NCSE & SE, RSE, SRSE & $200-400$ & $0-400$ & 81 & - \\
\hline $\begin{array}{l}\text { Koubeissi et al. } \\
\text { (2011) [59] }\end{array}$ & rs, sc & 4 & $53-79$ & NCSE & RSE & $50-200$ & $100-200$ & 100 & 0 \\
\hline $\begin{array}{l}\text { Rantsch et al. } \\
\text { (2011) [60] }\end{array}$ & rs, sc & 9 & $52-84$ & NCSE & RSE & $50-100$ & - & 20 & - \\
\hline $\begin{array}{l}\text { Jain and Harvey } \\
\text { (2011) [61] }\end{array}$ & $\mathrm{rs}, \mathrm{mc}$ & 31 & $12-17$ & CSE, NCSE & SE, RSE & $50-200$ & $0-400$ & $75-100$ & 4 \\
\hline $\begin{array}{l}\text { Cherry et al. (2012) } \\
\text { [62] }\end{array}$ & rs, sc & 24 & $24-80$ & NCSE, CSE & RSE & $100-400$ & $300-400$ & 38 & 31 \\
\hline $\begin{array}{l}\text { Mnatsakanyan et al. } \\
\text { (2012) [63] }\end{array}$ & rs, sc & 10 & $16-90$ & NCSE & RSE & $200-300$ & $200-400$ & 70 & - \\
\hline $\begin{array}{l}\text { Belcastro et al. } \\
\text { (2013) [64] }\end{array}$ & ps, sc & 16 & $77 \pm 7$ & NCSE & SE & 400 & 400 & 50 & - \\
\hline $\begin{array}{l}\text { Miró et al. (2013) } \\
\text { [65] }\end{array}$ & $\mathrm{ps}, \mathrm{mc}$ & 34 & $22-86$ & CSE, NCSE & RSE & $100-400$ & $100-600$ & 65 & 6 \\
\hline $\begin{array}{l}\text { Santamarina et al. } \\
\text { (2013) [66] }\end{array}$ & rs, sc & 31 & $21-85$ & CSE, NCSE & SE, RSE & $200-600$ & 400 & 85 & 13 \\
\hline $\begin{array}{l}\text { Sutter et al. (2013) } \\
\text { [67] }\end{array}$ & rs, sc & 86 & $65 \pm 15$ & CSE, NCSE & SE, RSE, SRSE & 200 & 200 & 93 & 0 \\
\hline $\begin{array}{l}\text { Legros et al. (2014) } \\
\text { [68] }\end{array}$ & $\mathrm{ps}, \mathrm{cs}$ & 25 & $36-74$ & CSE, NCSE & SE, RSE & $200-400$ & 400 & 36 & 20 \\
\hline $\begin{array}{l}\text { Kellinghaus et al. } \\
\text { (2014) [69] }\end{array}$ & rs, sc & 21 & $18-90$ & CSE, NCSE & RSE & $200-800$ & $200-800$ & 33 & - \\
\hline $\begin{array}{l}\text { Garcés et al. (2014) } \\
\text { [70] }\end{array}$ & $\mathrm{rs}, \mathrm{mc}$ & 55 & $18-90$ & CSE, NCSE & SE, RSE & $50-400$ & $50-400$ & 71 & 15 \\
\hline $\begin{array}{l}\text { Grosso et al. (2014) } \\
\text { [71] }\end{array}$ & $\mathrm{rs}, \mathrm{mc}$ & 11 & $3-16$ & CSE, NCSE & RSE & $6.7-9.9^{\mathrm{a}}$ & $8.8-13.9^{\mathrm{a}}$ & 45 & 0 \\
\hline $\begin{array}{l}\text { Poddar et al. (2016) } \\
\text { [72] }\end{array}$ & rs, sc & 9 & $0-16$ & CSE & SE, RSE & $3.3-10^{\mathrm{a}}$ & - & 78 & 11 \\
\hline $\begin{array}{l}\text { Moreno Morales } \\
\text { et al. (2015) [73] }\end{array}$ & ps, ob & 53 & $19-73$ & CSE, NCSE & SE, RSE, SRSE & 400 & 400 & 57 & - \\
\hline $\begin{array}{l}\text { d’Orsi et al. (2015) } \\
\text { [74] }\end{array}$ & $\mathrm{ps}, \mathrm{sc}$ & 23 & $33-85$ & CSE, NCSE & SE, RSE, SRSE & 200 & $200-400$ & 80 & 20 \\
\hline $\begin{array}{l}\text { Lang et al. (2016) } \\
\text { [75] }\end{array}$ & $\mathrm{ps}, \mathrm{mc}$ & 51 & $1-92$ & CSE, NCSE & SE, RSE, SRSE & 300 & $93-1200$ & 70 & 13 \\
\hline $\begin{array}{l}\text { Santamarina et al. } \\
\text { (2018) [76] }\end{array}$ & $\mathrm{rs}, \mathrm{mc}$ & 165 & av. 64 & NCSE & SE, RSE & $100-600$ & $200-400$ & 63 & 10 \\
\hline $\begin{array}{l}\text { Reif et al. (2018) } \\
\text { [77] }\end{array}$ & rs, sc & 1 & 28 & ASE & RSE & 400 & 400 & 100 & 0 \\
\hline $\begin{array}{l}\text { Ngampoopun et al. } \\
\text { (2018) [78] }\end{array}$ & $\mathrm{ps}, \mathrm{sc}$ & 11 & $7-16$ & NCSE & SE, RSE & 227 & 225 & 73 & 9 \\
\hline $\begin{array}{l}\text { Perrenoud et al. } \\
\text { (2017) [79] }\end{array}$ & $\mathrm{rs}, \mathrm{mc}$ & 40 & $34-88$ & - & SE, RSE & $100-800$ & - & 40 & - \\
\hline
\end{tabular}


Table 1 (continued)

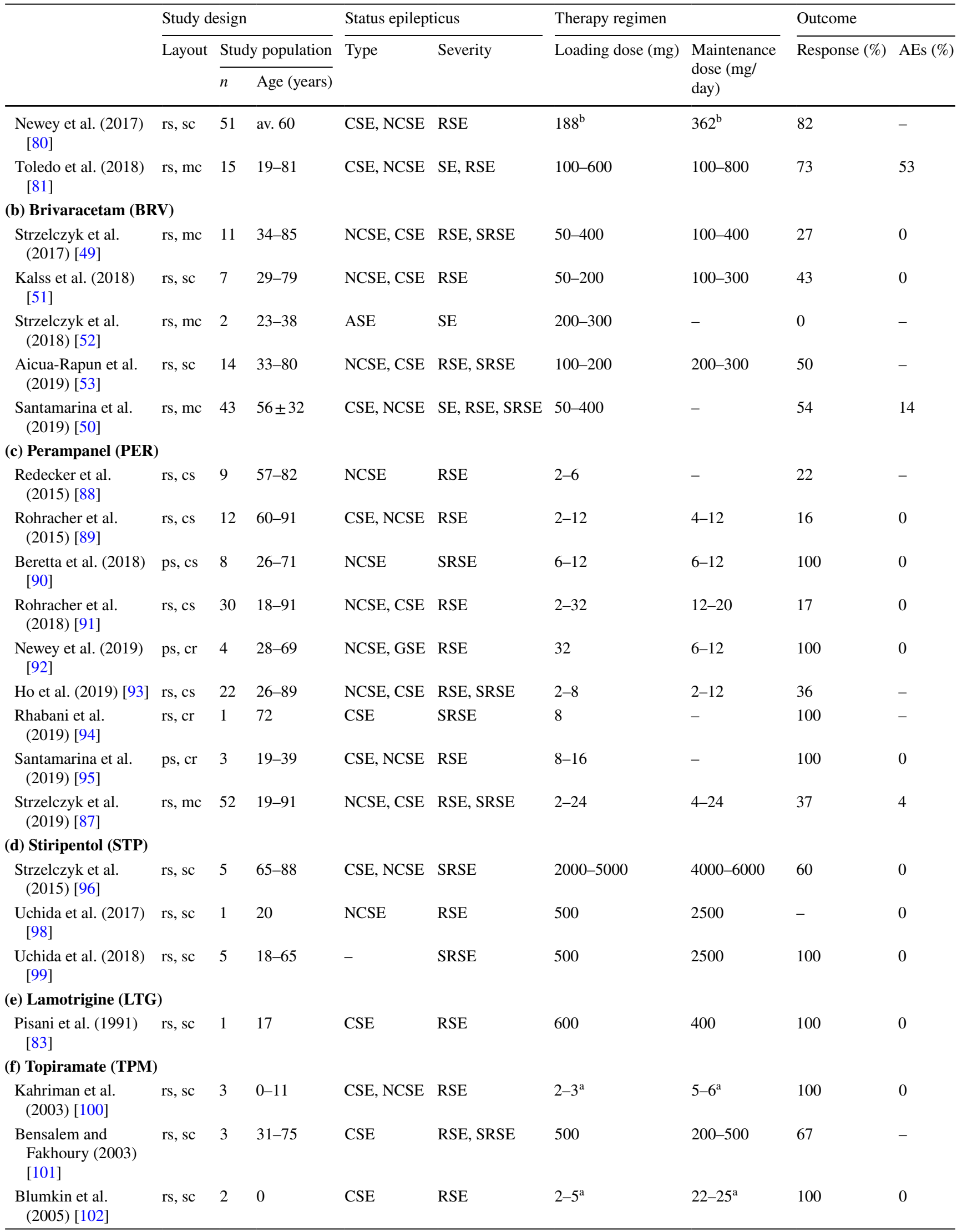


Table 1 (continued)

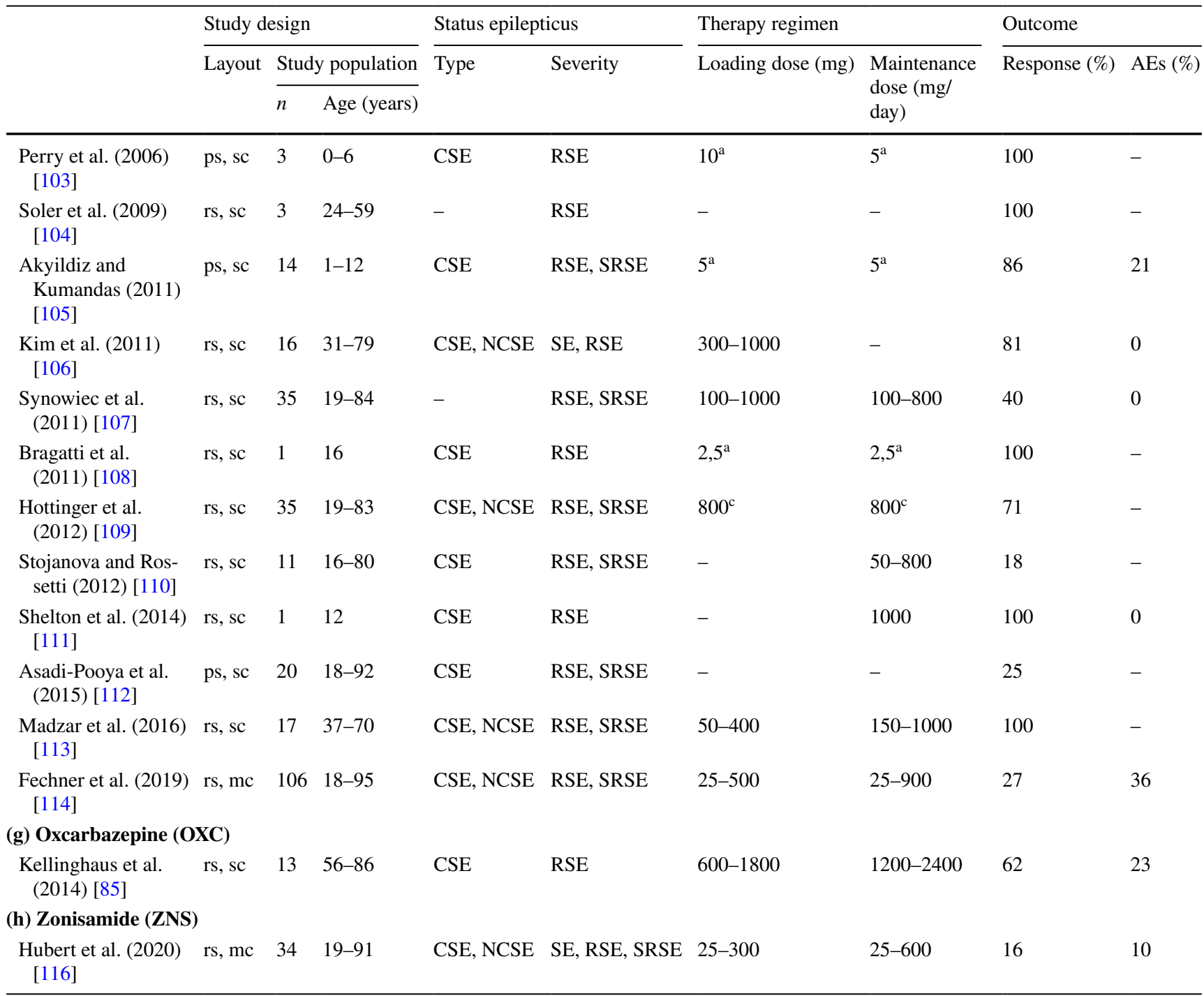

$A E s$ adverse events, $A S D s$ antiseizure drugs, $A S E$ absence SE, $a v$ average, $c s$ case series, $C S E$ convulsive SE, $c r$ case report, $m c$ multicenter, $N C S E$ nonconvulsive SE, $o b$ observational, $p s$ prospective, $r s$ retrospective, $R S E$ refractory SE, $s c$ single-center, $S E$ status epilepticus, $S R S E$ super-refractory SE

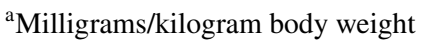

${ }^{\mathrm{b}}$ Average dose

${ }^{\mathrm{c}} \mathrm{Up}$ to

AEs have been reported in $0-77 \%$ of the enrolled patients, with bradycardia, hypotension, and relevant atrioventricular blockage as relevant substance-specific symptoms [55-81]. The largest retrospective multicenter cohort enrolled 165 patients with SE/RSE and reported a responder rate of $63 \%$ as well as good tolerability, with $10 \%$ of subjects reporting AEs [76]. Given this review focuses on patients in a PCS or with a LOT, one publication with a cohort of 15 adult patients with active cancer of various etiologies and SE/ RSE has to be highlighted. Here, a responder rate of 54\% and AEs in $14 \%$ of the enrolled subjects were reported [81].
According to the current state of knowledge, a loading dose of $5 \mathrm{mg} / \mathrm{kg}(200-400 \mathrm{mg})$ or greater and a maintenance dose of $200-400 \mathrm{mg} /$ day seem feasible (Table 1a).

LTG is a third-generation ASD approved for use in FE and genetic generalized epilepsy (GGE) as well as in Lennox-Gastaut syndrome, albeit available in oral tablet form only. Via the modulation of $\mathrm{Na}^{+}$channels, LTG suppresses the release of glutamate and aspartate as the dominant excitatory neurotransmitters. Based on its strong potential for interactions as well as the high risk of life-threatening skin reactions, including Stevens-Johnson syndrome, 
drug reaction with eosinophilia and systemic symptoms, and toxic epidermal necrolysis, LTG must be titrated slowly, usually requiring weeks to reach a therapeutic dosage [82]. Moreover, LTG has a high interaction potential with other drugs due to its exceptional hepatic metabolization. There is only one reported case of the successful use of LTG in RSE, from 1991 [83]. However, due to the mentioned aspects, there is currently no evidence supporting the efficacy, safety, or tolerability of LTG (Table 1e).

OXC is a third-generation ASD derivate of CBZ and a member of the dibenzazepine family approved for FE. OXC is a prodrug that is metabolized to its pharmacologically active derivate licarbazepine (MHD), which acts mainly by inactivating voltage-gated $\mathrm{Na}^{+}$channels. OXC is available as both a tablet and oral solution. Moreover, an anticonvulsive effect via enhanced potassium conductance and the modulation of voltage-gated calcium channels has been reported for this medication [84]. OXC is currently not approved for SE and there is only one retrospective case series with 13 enrolled adult patients supporting its efficacy, safety, and tolerability in RSE, with a responder rate of $62 \%$. AEs, especially hyponatremia, were reported in $23 \%$ of patients [85]. According to the current state of knowledge, a loading dose of $600 \mathrm{mg}$ and a maintenance dose of between 600 and $1800 \mathrm{mg} /$ day seem feasible (Table $1 \mathrm{~g}$ ).

PER is one of the newest established third-generation ASDs. It is approved as add-on therapy in FE and GGE, and is available as both a tablet and oral formulation (with an intravenous solution under development). PER acts as a noncompetitive antagonist at AMPA receptors [37]. Regarding the considerable glutamatergic proportion in the pathomechanism of SE [86], PER was granted special interest in the context of RSE and SRSE [37, 86, 87]. PER is not approved for use in SE but nine published case series and reports involving a total of 141 patients with CSE and NCSE are available that suggest a responder rate of 16-100\% [87-95]. The largest available retrospective, multicenter study with 53 adult patients reports a responder rate of $37 \%$ and good tolerability, with only sporadic AEs in 8\% (4/53) of the enrolled subjects [87]. According to the current state of knowledge, a loading dose of between 8 and $12 \mathrm{mg}$ (up to $32 \mathrm{mg}$ in few reported cases) and a maintenance dose of $10-12 \mathrm{mg} /$ day seem feasible (Table 1c).

STP is currently only approved for administration in patients with Dravet syndrome and refractory generalized tonic-clonic seizures as add-on therapy with VPA and clobazam, and is available only for oral application as a tablet or dissoluble granulate. STP acts mainly via activating the subunits $\alpha 3$ and $\delta$ of $\mathrm{GABA}_{\mathrm{A}}$ receptors. In addition, STP has been shown to increase extracellular GABA concentration via an unknown mechanism [96]. Being metabolized via the hepatic cytochrome P450 isoenzymes, STP secondarily increases the serum concentrations of many other ASDs, such as CBZ, VPA, PHT, and PB and many BZDs, supporting additional anticonvulsive properties [97]. There are three case reports or series in RSE/SRSE involving a total of 11 adult patients, revealing a responder rate of $0-100 \%$ as well as good tolerability of the treatment $[96,98,99]$. The largest case series includes five elderly patients between 65 and 88 years of age, and reports a responder rate of $60 \%$ without any AEs [96]. According to the current state of knowledge, a loading dose of 2000-4000 mg and a maintenance dose of 2000-3000 mg/day seem feasible. However, the comparably expensive costs of STP of approximately $€ 100$ ( $\$ 110$ or £85) per day have to be considered (Table 1d).

TPM is an orally available third-generation ASD approved for monotherapy and add-on therapy in FE and GGE that acts mainly via the modulation of voltage-gated $\mathrm{Na}^{+}$channels, and also via calcium channels, by increasing GABA levels and antagonism at the excitatory AMPA receptors. In both FE and GGE, TPM has been proven to be an effective and well-tolerated therapeutic option. TPM is not approved for use in SE. To date, there are 15 case reports and series with a total of 268 pediatric and adult subjects with RSE/SRSE and a responder rate of 17-100\% [100-114]. AEs (predominantly hyperammonemia) in seven reports (47\%), with a frequency of between 0 and $36 \%$, were reported, mostly during the simultaneous intake of VPA [100, 102, 105-107, 111, 114]. The largest retrospective multicenter study enrolled 106 adult patients and reported a responder rate of $27 \%$, as well as AEs in $36 \%$ of patients, additionally confirming the risk for relevant hyperammonemia in patients being simultaneously treated with VPA or PB [114]. Moreover, metabolic acidosis has been reported as a frequent $\mathrm{AE}$ [115]. According to the current state of knowledge, a loading dose of 100-200 $\mathrm{mg}$ and a maintenance dose of $200-600 \mathrm{mg} /$ day, or $2-5 \mathrm{mg} / \mathrm{kg}$ and $5 \mathrm{mg} / \mathrm{kg}$ in pediatric patients, seem feasible (Table 1f).

ZNS is a third-generation ASD approved as add-on therapy in FE with seizures with or without secondary generalization. ZNS is only available as an oral formulation and unfolds its anticonvulsive effect most likely via the modulation of voltage-gated $\mathrm{Na}^{+}$and T-type calcium channels. To date, only one study is available comprising 34 patients with SE using a median ZNS maintenance dose of $400 \mathrm{mg} /$ day after loading with a median dose of $100 \mathrm{mg}$. A clinical effect attributed to ZNS was observed in $16.2 \%$ of the SE patients, and possible AEs occurred in 10.4\% of the cohort [116].

\subsection{Other Minimally or Non-Invasive Therapeutic Options}

$\mathbf{M g S O}_{4}$ is a well-established prophylactic and therapeutic option in pregnant patients with pre-eclampsia and eclampsia-associated seizures; however, its use in non-eclamptic RSE is not approved [26]. Although its exact anticonvulsive 
mechanism of action is not well-understood at this time, $\mathrm{MgSO}_{4}$ is supposed to modulate and thereby inhibit $\mathrm{N}$-methyl D-aspartate (NMDA) receptors. Regarding its use in non-eclamptic RSE/SRSE, 23 case reports and case series involving a total of 32 adult and pediatric patients are available. A response to $\mathrm{MgSO}_{4}$ was reported in $38 \%$ (12/32) of patients, while $47 \%$ (7/15) showed a recurrence of seizures after discontinuation. AEs were addressed in four reports, including in detail in three of four subjects (75\%) [117-139]. In general, the use of $\mathrm{MgSO}_{4}$ has been controversially discussed by modern epileptologists; a bolus of $4 \mathrm{~g}$ followed by a continuous infusion of $2-6 \mathrm{~g} / \mathrm{h}$ is thought to increase the serum $\mathrm{Mg}$ level by approximately $3.5 \mathrm{mmol} / \mathrm{L}$ and has been suggested as appropriate (Table 2) [1,2,26].

The KD is a subtype of low-carb, high-fat and high-protein nutrition used as an established therapeutic approach in GLUT-1 deficiency and pyruvate dehydrogenase deficit, as well as refractory epilepsy. KD unfolds its anticonvulsive action by way of offering a limited neuronal energy supply, thereby prompting a general decrease in neuronal excitability. The presence of ketones in the blood and urine can be used as an indicator of a sufficient nutrition change. In the area of RSE and SRSE, 30 publications including a total of 157 pediatric and adult patients are available, using mainly KD in a $3: 1$ to $5: 1$ ratio $(27 / 30 ; 90 \%)[27,31,98$, $132,140-165]$. KD is usually initiated as an enteral solution but may also be induced by intravenous means [132]. Moreover, the sparse use of low glycemic index treatment, the adoption of medium-chain triglycerides, and additional use of an SGLT-2 inhibitor in a case of insufficient ketosis under KD have been reported (each 1/30; 3\%) [145, 160, 164]. Overall, $80 \%(125 / 157)$ of patients were responders and relevant AEs were observed in 47\% (55/117), including mostly gastroesophageal reflux, acidosis, or lipid/electrolyte unbalance. However, AEs were not addressed in all included publications (Table 3).

Neurosteroids with allopregnanolone (brexanolone) as the main representative of this class are endogenous derivates of progesterone acting mainly as positive allosteric modulators at the $\mathrm{GABA}_{\mathrm{A}}$ receptors. Allopregnanolone has

Table 2 Overview of the current evidence for the use of magnesium sulfate $\left(\mathrm{MgSO}_{4}\right)$ in refractory and super-refractory status epilepticus

\begin{tabular}{|c|c|c|c|c|c|c|c|c|c|}
\hline & \multicolumn{3}{|c|}{ Study design } & \multicolumn{2}{|c|}{ Status epilepticus } & \multicolumn{2}{|c|}{ Therapy regimen } & \multicolumn{2}{|l|}{ Outcome } \\
\hline & \multirow[t]{2}{*}{ Layout } & \multicolumn{2}{|c|}{$\begin{array}{l}\text { Study popula- } \\
\text { tion }\end{array}$} & \multirow[t]{2}{*}{ Type } & \multirow[t]{2}{*}{ Severity } & \multirow{2}{*}{$\begin{array}{l}\text { Duration of } \\
\text { treatment } \\
\text { (days) }\end{array}$} & \multirow{2}{*}{$\begin{array}{l}\text { Recurrence } \\
\text { after with- } \\
\text { drawal }\end{array}$} & \multirow[t]{2}{*}{ Response $(n / n)$} & \multirow[t]{2}{*}{ AEs $(n / n)$} \\
\hline & & $n$ & Age (years) & & & & & & \\
\hline Baxter et al. (2003) [117] & rs, sc & 1 & 6 & CSE & RSE & - & - & $0 / 1$ & - \\
\hline Berkley et al. (2015) [118] & rs, sc & 1 & 23 & CSE & RSE & - & - & $0 / 1$ & - \\
\hline Broomall et al. (2014) [119] & rs, sc & 1 & 11 & CSE & RSE & 4 & - & $0 / 1$ & - \\
\hline Dionisio et al. (2013) [120] & rs, sc & 1 & 17 & CSE & RSE & - & - & $0 / 1$ & - \\
\hline Fisher et al. (1988) [121] & rs, sc & 1 & 22 & CSE & RSE & 2 & $1 / 1$ & $0 / 1$ & $1 / 1$ \\
\hline Gedik et al. (2014) [122] & rs, sc & 1 & 5 & CSE & RSE & 4 & - & $0 / 1$ & - \\
\hline Nandakumar et al. (2008) [123] & rs, sc & 1 & 24 & CSE & RSE & - & - & $0 / 1$ & - \\
\hline Neligan et al. (2011) [124] & rs, sc & 1 & 33 & - & SE, RSE & - & $0 / 1$ & $1 / 1$ & - \\
\hline Pandey et al. (2010) [125] & rs, sc & 1 & 18 & CSE & RSE & 5 & - & $0 / 1$ & - \\
\hline Robakis and Hirsch (2006) [126] & rs, sc & 1 & 30 & CSE & RSE & 7 & - & $0 / 1$ & - \\
\hline Sadeh et al. (1991) [127] & rs, sc & 1 & 16 & CSE & RSE & 7 & $1 / 1$ & $1 / 1$ & $1 / 1$ \\
\hline Sahin et al. (2001) [128] & rs, sc & 2 & 13,15 & CSE & RSE & - & - & $1 / 2$ & - \\
\hline Savard et al. (2012) [129] & rs, sc & 1 & 27 & CSE & RSE & - & - & $0 / 1$ & - \\
\hline Shin et al. (2011) [130] & rs, sc & 1 & 57 & NCSE & RSE & 1 & $0 / 1$ & $1 / 1$ & - \\
\hline Storchheim (1933) [131] & rs, sc & 8 & - & - & SE, RSE & - & $4 / 8$ & $4 / 8$ & - \\
\hline Strzelczyk et al. (2013) [132] & rs, sc & 1 & 21 & CSE & RSE & 1 & - & $0 / 1$ & $1 / 1$ \\
\hline Tan et al. (2013) [133] & rs, sc & 1 & 2 & CSE & RSE & - & $0 / 1$ & $1 / 1$ & $0 / 1$ \\
\hline Valle-Morales et al. (2009) [134] & rs, sc & 1 & 29 & CSE & RSE & - & - & $0 / 1$ & - \\
\hline Visser et al. (2011) [135] & $\mathrm{rs}, \mathrm{sc}$ & 2 & 19,17 & CSE & RSE & 6 & $1 / 2$ & $2 / 2$ & - \\
\hline Madisia and Bergkeley (2014) [136] & $\mathrm{rs}, \mathrm{sc}$ & 1 & 23 & CSE & RSE & - & - & $0 / 1$ & - \\
\hline Zaatreh (2005) [137] & rs, sc & 1 & 48 & CSE & RSE & - & - & $1 / 1$ & - \\
\hline Hatch et al. (2016) [138] & rs, sc & 1 & 31 & CSE & RSE & - & - & $1 / 1$ & - \\
\hline Sahin and Riviello (2001) [139] & $\mathrm{rs}, \mathrm{sc}$ & 1 & 15 & CSE & RSE & - & - & $0 / 1$ & - \\
\hline
\end{tabular}

$A E s$ adverse events, $C S E$ convulsive SE, NCSE nonconvulsive SE, $r s$ retrospective, $R S E$ refractory SE, $s c$ single-center study, $S E$ status epilepticus 
Table 3 Overview of the current evidence for the use of a ketogenic diet in refractory and super-refractory status epilepticus

\begin{tabular}{|c|c|c|c|c|c|c|c|c|c|}
\hline & \multicolumn{3}{|c|}{ Study design } & \multicolumn{2}{|c|}{ Status epilepticus } & \multicolumn{2}{|c|}{ Therapy regimen } & \multicolumn{2}{|l|}{ Outcome } \\
\hline & \multirow[t]{2}{*}{ Layout } & \multicolumn{2}{|c|}{$\begin{array}{l}\text { Study popula- } \\
\text { tion }\end{array}$} & \multirow[t]{2}{*}{ Type } & \multirow[t]{2}{*}{ Severity } & \multirow[t]{2}{*}{ Diet type } & \multirow[t]{2}{*}{$\begin{array}{l}\text { Achieved level } \\
\text { of ketosis }(n / n)\end{array}$} & \multirow[t]{2}{*}{ Response $(n / n)$} & \multirow[t]{2}{*}{$\operatorname{AEs}(n / n)$} \\
\hline & & $\bar{n}$ & Age (years) & & & & & & \\
\hline Bodenant et al. (2008) [140] & $\mathrm{rs}, \mathrm{sc}$ & 1 & 54 & CSE & RSE & KD 4:1 & $1 / 1$ & & - \\
\hline Wusthoff et al. (2010) [141] & rs, sc & 2 & $29-34$ & CSE, NCSE & SRSE & KD 4:1 & $2 / 2$ & $2 / 2$ & - \\
\hline Nabbout et al. (2010) [142] & rs, sc & 9 & $0-6$ & CSE & RSE & KD 4:1 & $8 / 9$ & $7 / 9$ & - \\
\hline Cervenka (2011) [31] & rs, sc & 1 & 49 & FSE & SRSE & $\mathrm{KD} 4: 1$ & $1 / 1$ & $1 / 1$ & - \\
\hline Kramer et al. (2011) [143] & $\mathrm{rs}, \mathrm{mc}$ & 4 & $7-27$ & - & SRSE & - & - & $1 / 4$ & - \\
\hline Nam et al. (2011) [144] & ps, sc & 5 & $4-40$ & - & RSE & $\mathrm{KD} 4: 1$ & $5 / 5$ & $5 / 5$ & $5 / 5$ \\
\hline Martikainen et al. (2012) [145] & $\mathrm{rs}, \mathrm{sc}$ & 1 & 22 & CSE & SRSE & LGIT & - & $1 / 1$ & $0 / 1$ \\
\hline Vaccarezza et al. (2012) [146] & rs, sc & 5 & $1-14$ & CSE & SRSE & - & - & $4 / 5$ & - \\
\hline Sort et al. (2013) [147] & rs, sc & 3 & $3-11$ & CSE & SRSE & - & $3 / 3$ & $2 / 3$ & $1 / 3$ \\
\hline Strzelczyk et al. (2013) [132] & rs, sc & 1 & 26 & CSE & SRSE & KD 4:1 & $1 / 1$ & $1 / 1$ & - \\
\hline Caraballo et al. (2014) [148] & $\mathrm{rs}, \mathrm{sc}$ & 10 & $0-16$ & CSE & SRSE & KD 4:1 & $10 / 10$ & $7 / 10$ & $3 / 10$ \\
\hline Cobo et al. (2014) [149] & rs, sc & 4 & $0-13$ & CSE & SRSE & KD 4:1 & $4 / 4$ & $4 / 4$ & $1 / 4$ \\
\hline Thakur et al. (2014) [150] & $\mathrm{rs}, \mathrm{mc}$ & 10 & $23-51$ & - & SRSE & KD 3-4:1 & $9 / 10$ & $9 / 10$ & $3 / 10$ \\
\hline Amer et al. (2015) [151] & rs, sc & 1 & 21 & - & SRSE & $\mathrm{KD} 4: 1$ & $1 / 1$ & $1 / 1$ & - \\
\hline Caraballo et al. (2015) [152] & $\mathrm{rs}, \mathrm{sc}$ & 2 & $0-1$ & CSE & SRSE & KD 4:1 & $2 / 2$ & $2 / 2$ & $0 / 2$ \\
\hline Cash (2015) [153] & rs, sc & 1 & & CSE & SRSE & $\mathrm{KD} 4: 1$ & $1 / 1$ & $1 / 1$ & - \\
\hline Fung et al. (2015) [154] & $\mathrm{rs}, \mathrm{sc}$ & 4 & $8-16$ & CSE & SRSE & KD 4:1 & $3 / 4$ & $1 / 4$ & $2 / 4$ \\
\hline Lin et al. (2015) [155] & rs, sc & 1 & 6 & CSE & SRSE & KD 4:1 & $1 / 1$ & $1 / 1$ & \\
\hline Appavu et al. (2016) [27] & rs, sc & 10 & $2-16$ & CSE & SRSE & KD 4-5:1 & $9 / 10$ & $9 / 10$ & $1 / 10$ \\
\hline Caraballo et al. (2017) [156] & rs, sc & 6 & $2-9$ & CSE & RSE & KD 4:1 & - & $5 / 6$ & $0 / 6$ \\
\hline Cervenka et al. (2017) [157] & $\mathrm{ps}, \mathrm{mc}$ & 15 & $18-82$ & - & SRSE & $\mathrm{KD} 4: 1$ & $15 / 15$ & $11 / 15$ & $10 / 15$ \\
\hline Smith and Press (2017) [158] & $\mathrm{rs}, \mathrm{sc}$ & 9 & & & SRSE & - & $9 / 9$ & $8 / 9$ & $2 / 9$ \\
\hline Uchida et al. (2017) [98] & rs, sc & 1 & 20 & NCSE & RSE & - & - & $1 / 1$ & - \\
\hline Arya et al. (2018) [159] & $\mathrm{ps}, \mathrm{mc}$ & 14 & $0-19$ & - & RSE & $\mathrm{KD} 4: 1$ & $14 / 14$ & $10 / 14$ & $3 / 14$ \\
\hline Blunk et al. (2018) [160] & $\mathrm{rs}, \mathrm{sc}$ & 1 & 42 & CSE & RSE & KD/SGLT2 & $1 / 1$ & $0 / 1$ & $0 / 0$ \\
\hline Francis et al. (2019) [161] & rs, sc & 11 & $21-73$ & - & RSE & - & $10 / 11$ & $8 / 11$ & $10 / 11$ \\
\hline Park et al. (2019) [162] & rs, sc & 16 & $0-21$ & CSE & RSE & $3-4: 1$ & $14 / 16$ & $15 / 16$ & $10 / 16$ \\
\hline Peng et al. (2019) [163] & rs, sc & 7 & $1-13$ & - & RSE & $3-4: 1$ & $6 / 7$ & $7 / 7$ & $3 / 7$ \\
\hline Prasoppokakorn et al. (2019) [164] & rs, sc & 1 & 19 & CSE & SRSE & MCT KD & $1 / 1$ & $1 / 1$ & $0 / 1$ \\
\hline Noviawaty et al. (2020) [165] & $\mathrm{rs}, \mathrm{sc}$ & 1 & 38 & CSE & SRSE & $\mathrm{KD} 4: 1$ & $1 / 1$ & $1 / 1$ & \\
\hline
\end{tabular}

AEs adverse events, CSE convulsive SE, FSE febrile SE, KD ketogenic diet, LGIT low glycemic index treatment diet, $m c$ multicenter, $M C T$ KD medium-chain triglyceride ketogenic diet, NCSE nonconvulsive SE, $p s$ prospective, $r s$ retrospective, $R S E$ refractory SE, $s c$ single-center, $S E$ status epilepticus, $S G L T 2$ sodium-glucose co-transporter-2, SRSE super-refractory SE

faced high expectations as a new potential option to wean patients from anesthetic drugs during the treatment of RSE and SRSE. Three studies with a total of 29 patients have been published to date, showing promising results $[119,166$, 167]; however, a placebo-controlled RCT with 132 enrolled subjects (67 receiving verum vs. 65 receiving placebo) analyzing the safety and efficacy of allopregnanolone to wean from third-line ASDs in SRSE was aborted by the sponsor after preliminary results suggested no significant difference was present between the groups $(p=0.878)$. Within the RCT population, allopregnanolone was well-tolerated [168]. Moreover, the use of neurosteroids outside weaning of intravenous or inhalable anesthetic drugs has not been analyzed thus far.

\subsubsection{Other Immunomodulatory or Immunosuppressive Treatment Options (IT)}

Over the past decades, several ITs have been used in SE, such as SP, IVIGs or plasmapheresis/plasma exchange (PLEX). Based on their direct effects, these options have been classified as first-line ITs to allow a better differentiation from rituximab, cyclophosphamide, or other longterm immunosuppressants (second-line ITs). There were 72 
Table 4 Overview of the current evidence for the use of neurosteroids and immunomodulatory/immunosuppressive treatment in refractory and super-refractory status epilepticus

\begin{tabular}{|c|c|c|c|c|c|c|c|c|c|}
\hline & \multicolumn{3}{|l|}{ Study design } & \multicolumn{2}{|c|}{ Status epilepticus } & \multicolumn{3}{|c|}{ Therapy characteristics } & \multirow{3}{*}{$\begin{array}{l}\text { Outcome } \\
\text { Response }(n / n)\end{array}$} \\
\hline & \multirow[t]{2}{*}{ Layout (phase) } & \multicolumn{2}{|c|}{$\begin{array}{l}\text { Study popula- } \\
\text { tion }\end{array}$} & \multirow[t]{2}{*}{ Type } & \multirow[t]{2}{*}{ Severity } & \multirow[t]{2}{*}{ Duration (days) } & \multirow{2}{*}{$\begin{array}{l}\text { Loading } \\
\text { dose }(\mu \mathrm{g} / \\
\mathrm{kg} / \mathrm{h})\end{array}$} & \multirow[t]{2}{*}{ Rate $(\mu \mathrm{g} / \mathrm{kg} / \mathrm{h})$} & \\
\hline & & $n$ & Age (years) & & & & & & \\
\hline \multicolumn{10}{|c|}{ (a) Neurosteroids (allopregnanolone) } \\
\hline $\begin{array}{r}\text { Broomall et al. } \\
\text { (2014) [119] }\end{array}$ & rs, sc & 2 & 2,11 & CSE, NCSE & SRSE & 5 & - & $28-86$ & $\begin{array}{c}\text { Successful } \\
\text { weaning }\end{array}$ \\
\hline $\begin{array}{l}\text { Vaitkevicius } \\
\text { et al. (2017) } \\
\text { [167] }\end{array}$ & rs, sc & 2 & 23,28 & CSE, NCSE & SRSE & 5 & - & 86 & $\begin{array}{c}\text { Successful } \\
\text { weaning }\end{array}$ \\
\hline $\begin{array}{l}\text { Rosenthal et al. } \\
\text { (2017) [166] }\end{array}$ & $\mathrm{ps}, \mathrm{mc}(\mathrm{I} / \mathrm{II})$ & 25 & $10-76$ & - & SRSE & 5 & 286 & $86-156$ & $17 / 25$ \\
\hline $\begin{array}{c}\text { NCT02477618 } \\
(2019)[168]\end{array}$ & ps, mc (III) & 67 & 41.3 & CSE, NCSE & SRSE & 6 & 300 & $90-150$ & $\begin{array}{l}\text { Not superior } \\
\text { to placebo } \\
(p=0.878)\end{array}$ \\
\hline \multicolumn{10}{|c|}{ (b) Immunomodulatory/immunosuppressive treatment with SP, IVIG or PLEX } \\
\hline $\begin{array}{l}\text { Agan et al. } \\
\text { (2015) [199] }\end{array}$ & rs, sc & 1 & 19 & AI CSE & RSE & PLEX & & & $1 / 1$ \\
\hline $\begin{array}{l}\text { Agirre-Arri- } \\
\text { zubieta and } \\
\text { Moran (2012) } \\
{[200]}\end{array}$ & rs, sc & 1 & 28 & - & RSE & PLEX, IVMP & & & $0 / 1$ \\
\hline $\begin{array}{l}\text { Al-Ajlan et al. } \\
\text { (2014) [201] }\end{array}$ & rs, sc & 1 & 18 & AI CSE & RSE & SP, IVIG & & & $0 / 1$ \\
\hline $\begin{array}{l}\text { Alam et al. } \\
\text { (2013) [202] }\end{array}$ & rs, sc & 1 & 60 & AI CSE & RSE & \multicolumn{2}{|c|}{ SP, IVIG, PLEX, RTX } & & $0 / 1$ \\
\hline $\begin{array}{l}\text { Alam et al. } \\
\text { (2014) [203] }\end{array}$ & rs, sc & 1 & 20 & CSE & RSE & \multicolumn{2}{|l|}{ SP, IVIG } & & $0 / 1$ \\
\hline $\begin{array}{l}\text { Amer et al. } \\
\text { (2015) [151] }\end{array}$ & rs, sc & 1 & 21 & AI CSE & RSE & \multicolumn{2}{|l|}{ SP, IVIG, PLEX } & & $0 / 1$ \\
\hline $\begin{array}{l}\text { Armas et al. } \\
\text { (2013) [204] }\end{array}$ & rs, sc & 1 & 69 & & RSE & \multicolumn{2}{|l|}{ SP, IVIG, AZA } & & $1 / 1$ \\
\hline $\begin{array}{l}\text { Barnes et al. } \\
\text { (2013) [205] }\end{array}$ & rs, sc & 1 & 33 & CSE & RSE & \multicolumn{2}{|l|}{ SP, PLEX } & & $0 / 1$ \\
\hline $\begin{array}{l}\text { Bobb et al. } \\
\text { (2014) [206] }\end{array}$ & $\mathrm{rs}, \mathrm{sc}$ & 1 & 28 & - & RSE & \multicolumn{2}{|l|}{ IVIG } & & $1 / 1$ \\
\hline $\begin{array}{l}\text { Brigo et al. } \\
\text { (2018) [207] }\end{array}$ & rs, sc & 1 & 22 & AI CSE & RSE & \multicolumn{2}{|l|}{ SP } & & $1 / 1$ \\
\hline $\begin{array}{l}\text { Buenache et al. } \\
\text { (2012) [208] }\end{array}$ & rs, sc & 1 & 4 & FIRES & RSE & \multicolumn{2}{|l|}{ SP, IVIG, PLEX } & & $1 / 1$ \\
\hline $\begin{array}{l}\text { Buerger et al. } \\
\text { (2015) [209] }\end{array}$ & rs, sc & 1 & 74 & CSE & RSE & \multicolumn{2}{|l|}{ SP, PLEX } & & $0 / 1$ \\
\hline $\begin{array}{l}\text { Calabrace and } \\
\text { Witherspoon } \\
(2014)[210]\end{array}$ & $\mathrm{rs}, \mathrm{sc}$ & 1 & 19 & AI NCSE & RSE & \multicolumn{2}{|l|}{ SP, PLEX, RTX } & & $0 / 1$ \\
\hline $\begin{array}{l}\text { Caputo et al. } \\
\text { (2017) [211] }\end{array}$ & rs, sc & 1 & 13 & FIRES & RSE & \multicolumn{2}{|l|}{ SP, IVIG } & & $1 / 1$ \\
\hline $\begin{array}{l}\text { Caraballo et al. } \\
\text { (2013) [212] }\end{array}$ & rs, sc & 10 & - & FIRES & RSE & \multicolumn{2}{|c|}{ IVIG, PLEX, RTX } & & $3 / 10$ \\
\hline $\begin{array}{l}\text { Charles et al. } \\
\text { (2014) [213] }\end{array}$ & $\mathrm{rs}, \mathrm{sc}$ & 1 & 57 & NCSE & RSE & \multicolumn{2}{|l|}{ IVIG } & & $1 / 1$ \\
\hline $\begin{array}{l}\text { Chevret et al. } \\
\text { (2008) [214] }\end{array}$ & $\mathrm{rs}, \mathrm{sc}$ & 4 & $9^{\mathrm{a}}$ & FIRES & RSE & \multicolumn{2}{|l|}{ PLEX } & & $1 / 4$ \\
\hline $\begin{array}{l}\text { Chevret et al. } \\
\text { (2011) [215] }\end{array}$ & $\mathrm{rs}, \mathrm{sc}$ & 8 & $6^{\mathrm{a}}$ & FIRES & RSE & \multicolumn{2}{|l|}{ PLEX } & & $1 / 8$ \\
\hline
\end{tabular}


Table 4 (continued)

\begin{tabular}{|c|c|c|c|c|c|c|c|c|}
\hline & \multicolumn{3}{|l|}{ Study design } & \multicolumn{2}{|c|}{ Status epilepticus } & \multicolumn{2}{|l|}{ Therapy characteristics } & \multirow{3}{*}{$\begin{array}{l}\text { Outcome } \\
\text { Response }(n / n)\end{array}$} \\
\hline & \multirow[t]{2}{*}{ Layout (phase) } & \multicolumn{2}{|c|}{$\begin{array}{l}\text { Study popula- } \\
\text { tion }\end{array}$} & \multirow[t]{2}{*}{ Type } & \multirow[t]{2}{*}{ Severity } & \multirow[t]{2}{*}{ Duration (days) } & \multirow[t]{2}{*}{ Rate $(\mu \mathrm{g} / \mathrm{kg} / \mathrm{h})$} & \\
\hline & & $n$ & Age (years) & & & & & \\
\hline $\begin{array}{l}\text { Costas et al. } \\
\text { (2015) [216] }\end{array}$ & rs, sc & 1 & 5 & FIRES & RSE & IVIG, PLEX & & $0 / 1$ \\
\hline $\begin{array}{l}\text { Dishong et al. } \\
\text { (2013) [217] }\end{array}$ & rs, sc & 1 & 14 & FIRES & RSE & SP, IVIG, PLEX, Anakinra & & $0 / 1$ \\
\hline $\begin{array}{l}\text { Gall et al. } \\
\text { (2013) [218] }\end{array}$ & rs, sc & 2 & $30^{\mathrm{a}}$ & NORSE & RSE & PLEX, IVIG & & $2 / 2$ \\
\hline $\begin{array}{l}\text { Gedik et al. } \\
\text { (2014) [122] }\end{array}$ & $\mathrm{rs}, \mathrm{sc}$ & 1 & 5 & AI CSE & RSE & SP, PLEX & & $1 / 1$ \\
\hline $\begin{array}{l}\text { Ghamande } \\
\text { et al. (2013) } \\
\text { [219] }\end{array}$ & rs, sc & 1 & 41 & - & RSE & SP, IVIG, RTX & & $0 / 1$ \\
\hline $\begin{array}{l}\text { Gonzales et al. } \\
\text { (2011) [220] }\end{array}$ & rs, sc & 1 & 23 & NORSE & RSE & SP, IVIG & & $0 / 1$ \\
\hline $\begin{array}{l}\text { Hainsworth } \\
\text { et al. (2014) } \\
\text { [221] }\end{array}$ & rs, sc & 1 & 23 & AI CSE & RSE & IVIG, SP, PLEX, RTX & & $1 / 1$ \\
\hline $\begin{array}{l}\text { Hakimi et al. } \\
\text { (2014) [222] }\end{array}$ & rs, sc & 1 & 44 & AI CSE & RSE & SP, IVIG, PLEX & & $1 / 1$ \\
\hline $\begin{array}{l}\text { Hoang et al. } \\
\text { (2014) [223] }\end{array}$ & rs, sc & 1 & 40 & NCSE & RSE & IVIG, SP, PLEX & & $0 / 1$ \\
\hline $\begin{array}{l}\text { Howell et al. } \\
\text { (2012) [224] }\end{array}$ & rs, sc & 1 & 14 & FIRES & RSE & PLEX, RTX & & $0 / 1$ \\
\hline $\begin{array}{l}\text { Hribljan et al. } \\
\text { (2013) [225] }\end{array}$ & rs, sc & 4 & - & FIRES & RSE & SP, IVIG, PLEX & & $0 / 4$ \\
\hline $\begin{array}{l}\text { Incecik et al. } \\
\text { (2015) [226] }\end{array}$ & rs, sc & 1 & 16 & - & RSE & SP, IVIG, PLEX & & $0 / 1$ \\
\hline $\begin{array}{l}\text { Kaneko et al. } \\
\text { (2012) [227] }\end{array}$ & rs, sc & 1 & 17 & AI CSE & RSE & SP, IVIG, PLEX & & $1 / 1$ \\
\hline $\begin{array}{l}\text { Katsuse et al. } \\
\text { (2019) [228] }\end{array}$ & rs, sc & 1 & 48 & AI NCSE & RSE & SP & & $1 / 1$ \\
\hline $\begin{array}{l}\text { Khawaja et al. } \\
\text { (2014) [229] }\end{array}$ & rs, sc & 1 & 22 & AI CSE & RSE & IVIG, SP, PLEX, RTX & & $1 / 1$ \\
\hline $\begin{array}{l}\text { Khawaja et al. } \\
(2015) \text { [230] }\end{array}$ & rs, sc & 7 & $35^{\mathrm{a}}$ & AI/viral CSE & RSE & IVIG, SP, PLEX, RTX & & $0 / 7$ \\
\hline $\begin{array}{l}\text { Kirkpatrick } \\
\text { et al. (2011) } \\
\text { [231] }\end{array}$ & rs, sc & 1 & 19 & AI CSE & RSE & SP, PLEX, RTX & & $1 / 1$ \\
\hline $\begin{array}{l}\text { Kong et al. } \\
\text { (2018) [232] }\end{array}$ & rs, sc & 24 & $17^{\mathrm{a}}$ & AI CSE & RSE & SP, IVIG, PLEX, RTX, CP & & $20 / 24$ \\
\hline $\begin{array}{l}\text { Korff et al. } \\
\text { (2009) [233] }\end{array}$ & rs, sc & 1 & 9 & FIRES & RSE & SP, PLEX & & $0 / 1$ \\
\hline $\begin{array}{l}\text { Kramer et al. } \\
\text { (2011) [234] }\end{array}$ & $\mathrm{rs}, \mathrm{sc}$ & 1 & - & FIRES & RSE & SP, IVIG, PLEX & & $0 / 1$ \\
\hline $\begin{array}{l}\text { Labate et al. } \\
\text { (2013) [235] }\end{array}$ & rs, sc & 1 & 17 & AI CSE & RSE & SP, IVIG & & $1 / 1$ \\
\hline $\begin{array}{l}\text { Le Moigno } \\
\text { et al. (2014) } \\
\text { [236] }\end{array}$ & rs, sc & 1 & 6 & AI CSE & RSE & SP, PLEX, RTX & & $1 / 1$ \\
\hline $\begin{array}{l}\text { Lenoir et al. } \\
\text { (2012) [237] }\end{array}$ & rs, sc & 1 & 17 & AI CSE & RSE & SP, IVIG, PLEX & & $1 / 1$ \\
\hline $\begin{array}{l}\text { Li et al. (2013) } \\
\text { [238] }\end{array}$ & $\mathrm{rs}, \mathrm{sc}$ & 3 & $39,43,51$ & CSE, NCSE & RSE & SP, IVIG & & $2 / 3$ \\
\hline
\end{tabular}


Table 4 (continued)

\begin{tabular}{|c|c|c|c|c|c|c|c|c|}
\hline & \multicolumn{3}{|l|}{ Study design } & \multicolumn{2}{|c|}{ Status epilepticus } & \multicolumn{2}{|l|}{ Therapy characteristics } & \multirow{3}{*}{$\begin{array}{l}\text { Outcome } \\
\text { Response }(n / n)\end{array}$} \\
\hline & \multirow[t]{2}{*}{ Layout (phase) } & \multicolumn{2}{|c|}{$\begin{array}{l}\text { Study popula- } \\
\text { tion }\end{array}$} & \multirow[t]{2}{*}{ Type } & \multirow[t]{2}{*}{ Severity } & \multirow[t]{2}{*}{$\begin{array}{ll}\text { Duration (days) } & \begin{array}{l}\text { Loading } \\
\text { dose }(\mu \mathrm{g} /\end{array}\end{array}$} & \multirow[t]{2}{*}{ Rate $(\mu \mathrm{g} / \mathrm{kg} / \mathrm{h})$} & \\
\hline & & $n$ & Age (years) & & & & & \\
\hline $\begin{array}{l}\text { Lin et al. } \\
\text { (2018) [239] }\end{array}$ & rs, sc & 45 & $1-18$ & CSE & RSE & SP, IVIG vs. IVIG & & $10 / 45$ \\
\hline $\begin{array}{l}\text { Lousa et al. } \\
\text { (2000) [240] }\end{array}$ & rs, sc & 1 & 14 & - & RSE & PLEX & & $1 / 1$ \\
\hline $\begin{array}{l}\text { Madisi and } \\
\text { Berkley } \\
\text { (2014) [241] }\end{array}$ & $\mathrm{rs}, \mathrm{sc}$ & 1 & 23 & NORSE, CSE & RSE & IVIG, SP, PLEX, RTX & & $1 / 1$ \\
\hline $\begin{array}{l}\text { Malaga et al. } \\
\text { (2015) [242] }\end{array}$ & $\mathrm{rs}, \mathrm{sc}$ & 1 & 1 & AI CSE & RSE & SP, IVIG, PLEX, RTX & & $1 / 1$ \\
\hline $\begin{array}{l}\text { Mann et al. } \\
\text { (2013) [243] }\end{array}$ & rs, sc & 1 & 20 & AI CSE & RSE & IVIG, SP, PLEX & & $1 / 1$ \\
\hline $\begin{array}{l}\text { Marques et al. } \\
\text { (2014) [244] }\end{array}$ & rs, sc & 1 & 30 & AI CSE & RSE & SP, IVIG & & $1 / 1$ \\
\hline $\begin{array}{l}\text { Milh et al. } \\
\text { (2012) [245] }\end{array}$ & rs, sc & 1 & 5 & AI CSE & RSE & IVIG & & $1 / 1$ \\
\hline $\begin{array}{l}\text { Mirás Veiga } \\
\text { et al. (2016) } \\
\text { [246] }\end{array}$ & rs, sc & 1 & 4 & FIRES & RSE & SP, PLEX, IVIG & & $0 / 1$ \\
\hline $\begin{array}{l}\text { Moeller et al. } \\
\text { (2012) [247] }\end{array}$ & rs, sc & 1 & 27 & AI CSE & RSE & IVIG, PLEX & & $0 / 1$ \\
\hline $\begin{array}{l}\text { Nakamura } \\
(2015) \text { [248] }\end{array}$ & rs, sc & 1 & 1 & FIRES & RSE & SP, IVIG, PLEX & & $0 / 1$ \\
\hline $\begin{array}{l}\text { Neligan et al. } \\
\text { (2011) [124] }\end{array}$ & rs, sc & 1 & 33 & AI CSE & RSE & IVIG, RTX & & $1 / 1$ \\
\hline $\begin{array}{l}\text { Noviawaty } \\
\text { et al. (2015) } \\
\text { [249] }\end{array}$ & rs, sc & 1 & 13 & AI CSE & RSE & PLEX & & $0 / 1$ \\
\hline $\begin{array}{l}\text { Ogawa et al. } \\
\qquad(2013) \text { [250] }\end{array}$ & rs, sc & 1 & 11 & AERRPS & RSE & PLEX & & $0 / 1$ \\
\hline $\begin{array}{l}\text { Pari et al. } \\
\text { (2014) [251] }\end{array}$ & rs, sc & 1 & 19 & AI NCSE & RSE & SP, PLEX & & $1 / 1$ \\
\hline $\begin{array}{l}\text { Ramos et al. } \\
\text { (2019) [169] }\end{array}$ & rs, sc & 4 & $51-75$ & CSE, NCSE & RSE & SP & & $4 / 4$ \\
\hline $\begin{array}{l}\text { Rypulak et al. } \\
\text { (2016) [252] }\end{array}$ & rs, sc & 1 & 23 & AI CSE & RSE & IVIG, SP, & & $1 / 1$ \\
\hline $\begin{array}{l}\text { Sawicka et al. } \\
\text { (2016) [253] }\end{array}$ & rs, sc & 1 & 18 & AI CSE & RSE & IVIG, PLEX, RTX & & $1 / 1$ \\
\hline $\begin{array}{l}\text { Shatzmiller } \\
\text { et al. (2011) } \\
\text { [254] }\end{array}$ & rs, sc & 1 & 19 & AI CSE & RSE & SP, IVIG, PLEX & & $1 / 1$ \\
\hline $\begin{array}{l}\text { Shrivastava } \\
\text { et al. (2017) } \\
\text { [255] }\end{array}$ & rs, sc & 1 & 24 & NORSE & RSE & SP, IVIG, PLEX & & $1 / 1$ \\
\hline $\begin{array}{l}\text { Soldatos and } \\
\text { Gorman } \\
\text { (2012) [256] }\end{array}$ & rs, sc & 1 & 6 & FIRES & RSE & SP, IVIG, PLEX & & $1 / 1$ \\
\hline $\begin{array}{l}\text { Thomas et al. } \\
\text { (2012) [257] }\end{array}$ & rs, sc & 1 & 19 & AI NCSE & RSE & SP, IVIG, PLEX & & $1 / 1$ \\
\hline $\begin{array}{l}\text { Ting et al. } \\
\text { (2017) [258] }\end{array}$ & rs, sc & 1 & 16 & AI CSE & RSE & SP, PLEX, CP & & $1 / 1$ \\
\hline $\begin{array}{l}\text { Triplet et al. } \\
\text { (2018) [259] }\end{array}$ & rs, sc & 1 & 21 & AI CSE & RSE & SP, IVIG, PLEX, RTX, CP & & $1 / 1$ \\
\hline
\end{tabular}


Table 4 (continued)

\begin{tabular}{|c|c|c|c|c|c|c|c|c|c|}
\hline & \multicolumn{3}{|l|}{ Study design } & \multicolumn{2}{|c|}{ Status epilepticus } & \multicolumn{3}{|c|}{ Therapy characteristics } & \multirow{3}{*}{$\begin{array}{l}\text { Outcome } \\
\text { Response }(n / n)\end{array}$} \\
\hline & \multirow[t]{2}{*}{ Layout (phase) } & \multicolumn{2}{|c|}{$\begin{array}{l}\text { Study popula- } \\
\text { tion }\end{array}$} & \multirow[t]{2}{*}{ Type } & \multirow[t]{2}{*}{ Severity } & \multirow[t]{2}{*}{ Duration (days) } & \multirow{2}{*}{$\begin{array}{l}\text { Loading } \\
\text { dose }(\mu \mathrm{g} / \\
\mathrm{kg} / \mathrm{h})\end{array}$} & \multirow[t]{2}{*}{ Rate $(\mu \mathrm{g} / \mathrm{kg} / \mathrm{h})$} & \\
\hline & & $n$ & Age (years) & & & & & & \\
\hline $\begin{array}{l}\text { Van Baalen } \\
\text { et al. }(2012) \\
\text { [260] }\end{array}$ & rs, sc & 6 & $2-12$ & FIRES & RSE & SP, IVIG, PLEX & & & $1 / 6$ \\
\hline $\begin{array}{l}\text { Villani et al. } \\
\text { (2001) [261] }\end{array}$ & rs, sc & 1 & 45 & CSE & RSE & SP, IVIG, PLEX & & & $1 / 1$ \\
\hline $\begin{array}{l}\text { Wilder-Smith } \\
\text { et al. (2005) } \\
\text { [262] }\end{array}$ & rs, sc & 3 & - & NORSE, CSE & RSE & IVIG & & & $0 / 1$ \\
\hline $\begin{array}{l}\text { Yamamoto } \\
\text { et al. (2014) } \\
\text { [263] }\end{array}$ & $\mathrm{rs}, \mathrm{sc}$ & 1 & 35 & AI CSE & RSE & SP, IVIG, PLEX & & & $1 / 1$ \\
\hline $\begin{array}{l}\text { Yeo et al. } \\
\text { (2009) [264] }\end{array}$ & rs, sc & 1 & 32 & AI CSE & RSE & IVIG & & & $1 / 1$ \\
\hline $\begin{array}{l}\text { Yoshida et al. } \\
\text { (2019) [265] }\end{array}$ & rs, sc & 1 & 68 & PNS NCSI & RSE & SP, PLEX & & & $1 / 1$ \\
\hline
\end{tabular}

AERRPS acute encephalitis with refractory repetitive partial seizures, $A I$ autoimmune, $A Z A$ azathioprine, $C P$ cyclophosphamide, $C S E$ convulsive SE, FIRES febrile infection-related epilepsy syndrome, IVIG intravenous immunoglobulins, $m c$ multicenter study, $N C S E$ non-convulsive SE, NORSE new-onset refractory status epilepticus, PLEX plasma exchange or plasmapheresis, PNS paraneoplastic neurological syndrome, ps prospective, $r s$ retrospective, $R S E$ refractory SE, $R T X$ rituximab, $s c$ single-center, $S E$ status epilepticus, $S P$ steroid pulse, $S R S E$ super-refractory SE

${ }^{\text {a Median }}$

studies with 175 patients who used first-line ITs, of which $14(19 \%)$ used a single therapy regimen, $20(28 \%)$ used a double therapy regimen, and $38(53 \%)$ used a triple or quadruple therapy regimen. Overall, $46 \%(n=81)$ of the patients treated with IT showed an attributable response. The data situation was insufficient to allow for analyzing the AEs. For more information on the included studies, please refer to Table 4.

\section{Discussion}

Based on a survey of existing publications, no level 1, grade A evidence is available for the use of any of the reviewed fourth-line ASDs in RSE. The best data were found for LCM and TPM, with a level 3 evidence and grade $C$ recommendation. Moreover, there was level 4, grade D evidence for the use of BRV and PER, or level 5, grade D evidence for STP, $\mathrm{OXC}$, and ZNS in RSE. Regarding the other assessed minimally or non-invasive, non-medicinal therapeutic options, level 4, grade D evidence supporting the benefit of the KD, and level 5, grade D evidence supporting the use of $\mathrm{MgSO}_{4}$, in the context of non-eclamptic RSE, were available. The evidence for ITs, such as SP, IVIG, or PLEX, was level 4, grade D, with the additional limitation that most studies showing an effect of ITs included patients with autoimmune
SE. Even if single studies supported the hypothesis, the SP use could be a feasible option as an abortive treatment for refractory seizures or status epilepticus [169], but is no reliable evidence for their broad use in RSE in general [170]. There were contradictory results and no reliable evidence regarding the use of neurosteroids in RSE, with the evidence formally rated as level 5, grade D (Table 4). Based on these results, the use of neurosteroids in RSE treatment cannot be recommended (Table 4), although studies of ganaxolone in humans are in progress [171]. All mentioned medicinal or non-medicinal therapeutic options do not require a stay in the ICU or other invasive measures beyond demand-oriented continuous monitoring that is usually affected by ACP or LOT, or within a moderate PCS. Regarding PLEX, establishing a central venous catheter, as well as the often-exhausting experience of therapeutic cycles, has to be reflected upon and discussed in regard to the individual advance directives. Nearly all reviewed therapy options can be applied orally or enterally via a nasogastric tube, which underlines their applicability in patients with impairment of consciousness as well as dysphagia or aphagia. Due to rarely reported cardiac arrhythmia or de novo atrioventricular blockage [26], $\mathrm{MgSO}_{4}$ should be applied intravenously for improved controllability and to prepare for the possibility of rapid discontinuation in the case of the above-mentioned symptoms. Due to the obligatory need for advanced cardiopulmonary 
monitoring of established anesthetic drugs, such as ketamine, propofol, thiopental or midazolam, in low doses and not requiring mechanical ventilation, they were not included in this systematic review but may also be individually considered $[2,172,173]$. For the same reasons, sporadically used neuromodulation techniques in RSE, such as vagal nerve stimulation (VNS), electroconvulsive therapy (ECT), transcranial magnetic stimulation (TMS), and deep brain stimulation (DBS) were also not included in this review $[174,175]$.

As with any systemic review, this publication may suffer from several methodical limitations, such as possible missing relevant publications not identified by the prescribed search algorithm or the cross-check via pre-existing reviews on this topic. Moreover, the exclusion of articles not available in the English, French, German, Italian, or Spanish languages, as well as the limitation of the research on the MEDLINE, EMBASE and Cochrane databases, represents possible biases of this review. In particular, all studies on RSE and SRSE are subject to bias due to consecutive addon therapy with several ASDs or other therapeutic options, which makes it difficult to link a specific effect to one of the used interventions. Most included publications try to solve this bias by temporal correlation of therapy onset and the observed effect; however, this established way of analysis does not exclude the occurrence of a prolonged effect of previously used interventions or a cumulative effect of different therapeutic approaches [176, 177]. To minimize the mentioned limitations and to offer a structured analysis, PRISMA guidelines were closely followed [178].

The management of RSE in patients with a LOT or a PCS represents a challenge for modern clinicians, intensive care medics, and epileptologists. Due to patients' advanced directives or specific health conditions, recommendations of existing guidelines have to be frequently omitted as invasive treatment in the ICU may be refused or individual contraindications for specific second- or third-line treatment options exist. In the case of a sustained wish for a conservative therapeutic attempt, clinicians have to fall back on fourth-line ASDs as the last therapeutic resort. The evidence for the use of fourth-line ASDs in RSE and SRSE is limited; however, there are several therapeutic options that have been shown to be effective, safe, and well-tolerated whose use can be translated in the context of ACP, LOT, and PCS. In accordance with frequent ACP, LOT or PCS, all ASDs as well as KD can be applied enterally without the need to adopt invasive measures such as a central venous catheter or port catheter. In all patients, basal therapy with BZDs should be initiated if possible [19, 179]. The highest level of evidence in RSE/ SRSE exists to support the use of LCM and TPM (level 3, grade $\mathrm{C}$ ), which have been proven to be effective and welltolerated in patients with RSE [36, 114]. Moreover, there is sufficient evidence for the use of PER (level 4, grade D) [87],
BRV (level 4, grade D), OXC (level 5, grade D) [85], ZNS (level 5, grade D) and STP (level 5, grade D) [96] in patients with RSE or SRSE (Table 1). Here, the reduced potential for psychobehavioral adverse effects, as well as the availability of an intravenous formulation for BRV and the pleiotropic effects of STP, have to be mentioned [37, 96]. The use of LTG, ESL, and CBZ cannot be recommended based on the current state of knowledge. In line with conventional clinical experience, data from rodent studies suggested that early and aggressive combination therapy in RSE treatment is beneficial, which was attributed to an early maladaptive internalization of synaptic $\mathrm{GABA}_{\mathrm{A}}$ receptors and the externalization of NMDA receptors [180]. Here, the unique mode of action of LEV, BRV, PER or STM could be of particular advantage, which has to be further elucidated by future trials and research work $[37,96]$. However, even if these aspects should not be the focus of the therapy decision, economic aspects of different ASD may also have to be considered [181, 182].

Considering non-medicinal therapeutic options, there is low evidence for the use of KD (level 4, grade D) in RSE based on more than 150 reported cases. This therapy should be started and monitored by a physician experienced in $\mathrm{KD}$, or a nutritional expert, to avoid electrolyte or vitamin imbalance or hypoglycemia, and standardized protocols should be followed [157]. A recent study on the nutritional state in SE showed that the induction of ketogenesis might improve treatment outcomes and will surely stimulate the conduct of further research into the adoption of KD in SE [183]. The use and benefits of $\mathrm{MgSO}_{4}$ in non-eclamptic RSE are controversially discussed within the field $[26,184]$ and are only supported by weak evidence (level 5 , grade D). There is currently no evidence available for the use of neurosteroids in RSE, and, furthermore, there is even evidence against their use [168]. The use of immunotherapies such as steroids, plasmapheresis, or IVIGs also cannot be recommended unless an autoimmune or paraneoplastic etiology is proven or seems obvious $[185,186]$.

Following the GRADE classification, the authors of this manuscript came to a consensus for a 'high' recommendation for the use of LCM, TPM, and BRV; a 'moderate' recommendation for the use of PER and STM; a 'low' recommendation for the use of OXC, ZNS, and $\mathrm{KD}$; and a 'very low' recommendation for the use of $\mathrm{MgSO}_{4}$ and ITs in patients without presumptive autoimmune etiology of SE. The level of evidence, grade of recommendation, feasible loading and maintenance doses, route of application, and relevant AEs for every mentioned therapeutic option are given in Table 5.

In otherwise healthy patients with RSE/SRSE, rational diagnostics tests should be performed to identify the possible causes or factors leading to disease persistence. According to the German clinical practice guidelines on SE, routine 
Table 5 Feasible non- or minimally invasive therapeutic options for RSE in patients undergoing LOT or in a PCS, beyond current recommendations of existing guidelines

\begin{tabular}{|c|c|c|c|c|c|c|c|c|}
\hline \multicolumn{2}{|l|}{ Substance } & \multicolumn{2}{|c|}{ Level of evidence } & \multicolumn{2}{|l|}{ Dosing } & \multicolumn{2}{|c|}{$\begin{array}{l}\text { ApplicationAppli- } \\
\text { cation }\end{array}$} & \multirow[t]{2}{*}{$\begin{array}{l}\text { Important treatment } \\
\text { issues }\end{array}$} \\
\hline Name & INN & Oxford [42] & GRADE [43] & Loading (mg) & Maintenance (mg/day) & Regimen & Route & \\
\hline \multicolumn{9}{|l|}{ Antiseizure drugs } \\
\hline Topiramate $^{\mathrm{a}}$ & TPM & $3 \mathrm{C}$ & I & 400 & $400-600$ & bid & po/os & $\begin{array}{l}\text { Hyperammonemia, } \\
\text { metabolic acidosis }\end{array}$ \\
\hline Brivaracetam $^{\mathrm{a}}$ & BRV & $4 \mathrm{D}$ & I & $2^{\mathrm{b}}$ & $100-200$ & bid & po/os/iv & $\begin{array}{l}\text { Psychobehavioral } \\
\text { changes }\end{array}$ \\
\hline Perampanel $^{\mathrm{a}}$ & PER & $4 \mathrm{D}$ & II & $8-12$ & $8-12$ & $\mathrm{qd}$ & po/os & Dizziness, vertigo \\
\hline Lacosamide $^{\mathrm{a}}$ & LCM & $3 \mathrm{C}$ & I & $5^{\mathrm{b}}$ & $200-400$ & bid & po/os/iv & $\begin{array}{l}\text { AV block } \geq^{\circ} \mathrm{III} \\
\text { relevant bradycardia }\end{array}$ \\
\hline Stiripentol $^{\mathrm{a}}$ & STP & $5 \mathrm{D}$ & II & $2000-3000$ & $3000-4000$ & bid & po/os & $\begin{array}{l}\text { High daily treatment } \\
\text { costs }\end{array}$ \\
\hline Oxcarbazepine $^{\mathrm{a}}$ & $\mathrm{OXC}$ & $5 \mathrm{D}$ & III & $600-1200$ & $1200-1800$ & bid & po/os & Hyponatremia \\
\hline Zonisamide $^{\mathrm{a}}$ & ZNS & $5 \mathrm{D}$ & III & $200-300$ & $200-600$ & bid & po/os & Ataxia, skin rash \\
\hline \multicolumn{9}{|c|}{ Other minimally or non-invasive therapeutic options } \\
\hline Magnesium sulfate & $\mathrm{MgSo} 4$ & $5 \mathrm{D}$ & IV & 4000 & $2000-6000^{c}$ & cont & iv & Cardiac arrhythmia \\
\hline Ketogenic diet ${ }^{\mathrm{a}}$ & $\mathrm{KD}$ & $4 \mathrm{D}$ & III & KD 4:1 ratio & $\mathrm{KD} 4: 1$ & cont & po & $\begin{array}{l}\text { Acidosis, lipid or } \\
\text { electrolyte disbal- } \\
\text { ance, GER }\end{array}$ \\
\hline Steroid pulse ${ }^{a}$ & IVMP & $4 \mathrm{D}$ & III & $1000-2000$ & $1000-2000$ & qd & iv & $\begin{array}{l}\text { Only if autoimmune } \\
\text { etiology is assumed } \\
\text { or confirmed }\end{array}$ \\
\hline Immunoglobulins $^{\mathrm{a}}$ & IVIG & $4 \mathrm{D}$ & III & $2^{\mathrm{d}}$ & & tid & iv & $\begin{array}{l}\text { Only if autoimmune } \\
\text { etiology is assumed } \\
\text { or confirmed }\end{array}$ \\
\hline Plasma exchange $^{\mathrm{a}}$ & PLEX & $4 \mathrm{D}$ & III & NA & NA & daily & iv & $\begin{array}{l}\text { Only if autoimmune } \\
\text { etiology is assumed } \\
\text { or confirmed }\end{array}$ \\
\hline
\end{tabular}

$A V$ atrioventricular, bid twice daily, Cont. continuous application, GER gastroesophageal reflux, GRADE Grading Recommendations Assessment, Development, and Evaluation, $i v$ intravenous, IVMP intravenous methylprednisolone, KD ketogenic diet, LOT limitation of life-sustaining therapy, $N A$ not applicable, $o s$ oral solution available, po orally, PCS palliative care setting, $q d$ once daily, $R S E$ refractory status epilepticus, tid three times daily

${ }^{a}$ Use and dose recommendations not in line with the official approval

${ }^{\mathrm{b}}$ Milligram/kilogram body weight

${ }^{\mathrm{c}} \mathrm{Grams} /$ hour as a continuous infusion

${ }^{\mathrm{d}}$ Grams/kilogram body weight, distributed over 2-5 days

point-of-care and laboratory testing (including blood glucose, hemography, inflammation parameters, electrolytes, liver, pancreas, renal and thyroid function, and creatine kinase) and cerebral imaging (e.g. computed tomography, magnetic resonance imaging) should be performed, supplemented by lumbar puncture where appropriate. Possible causal, maintenance or trigger factors for RSE/SRSE should be addressed and treated adequately as long as the therapy administered is consistent with the patient's ACP, LOT, or PCS $[5,187]$.

More so than in any other population, ACP, advance directives, and medical ethical aspects have to be considered carefully in these patients before the initiation of therapy, and should be re-evaluated during the course of treatment.
According to the literature, only a small portion (4.6-39.0\%) of patients with SE or other critical neurological diseases had written advance directives or directives disclosed by designated healthcare agents (also known as healthcare proxy, i.e. a person who can act and decide on medical matters if you are not able to do so yourself), which were mostly effecting a do-not-resuscitate (DNR) order [188-190]. Here, the availability of an informed healthcare agent was associated with a slightly increased proportion of patients with DNR orders. Advance directives are largely accepted by physicians regarding both the adaption of the therapy setting and the withholding of resuscitation [190].

Realistic expectations of the outcome of treatment and the timely anticipation of a further disease course are warranted. 
In this context, the potential harm of prolonged iatrogenic coma and its secondary risks due to supportive aids and medications (e.g. vasopressors, paralysis of the bowels) that have been previously shown in elderly and multimorbid patients has to be addressed [190-193]. Referring to the written advance directive directly or indirectly (through conversation) will guide the therapeutic approach, with treatment steps checked for their compatibility and discussed with the patient, caregivers, or healthcare agents. For example, before introducing artificial enteral feeding via a nasogastric tube or intravenously as an indispensable step for the $\mathrm{KD}$, it should be considered whether the patient would have agreed to this procedure and artificial nutrition itself, and whether the resulting decrease in quality of life due to both measures would have been accepted. In the case of refusal, the probability for reaching ketosis over the stay as a consequence of catabolic metabolism is also high [194], but less controllable and of unclear therapeutic relevance. Moreover, fundamental therapeutic aspects, such as the renunciation of resuscitation or the immediate switching to a supportive care setting in the case of acute deterioration, should be addressed early if they were not already mentioned in the ACP documentation. In this context, the withdrawal and withholding of life-supporting treatment, including ASDs, as a possible measure of LOT should also be addressed [188-190]. A reevaluation of the health condition, therapeutic approaches, and further diagnostic or therapeutic steps should be performed at regular intervals and discussed with the patient, their caregivers or their healthcare agents where appropriate. If any doubts exist as to the medical ethical tenability of the chosen therapy regimen and the patient's advance directive, a third neutral party, such as an ethics committee should be consulted. In addition, the well-being and possible illnessspecific consequences of caregivers should be addressed during and after therapy [195].

The approximate mortality rate for RSE and SRSE in unrestricted therapy settings is 35-40\% [12, 196-198], supporting such an outcome in a far higher proportion of patients with ACP, undergoing LOT or in a PCS. In the case of a continuing refractory course or an acute deterioration of the health state, palliative sedation and analgesia using BZDs, propofol, or morphine should be considered within the setting of a dying patient, with the aim of anxiolysis, freedom from pain and convulsive seizures, and mitigation of respiratory distress $[18,19]$.

\section{Conclusion}

Refractory SE in patients undergoing LOT or in a PCS represents a difficult challenge for modern clinicians, intensivists and epileptologists. The evidence for the use of ASDs and other minimally or non-invasive therapy options in RSE beyond that in current guidelines is low, but several effective and well-tolerated therapies are available that should be considered in this patient population. In particular, newer thirdand fourth-generation ASDs such as LCM, TPM, BRV and PER seem to be a feasible escalation option for the treatment of RSE in patients undergoing LOT or in a PCS. These drugs can be rapidly uptitrated and applied orally, via nasogastric tube, and two of them are also available as an intravenous solution. In addition, the KD represents a practicable noninvasive therapy option that may be considered individually. More so than in any other population, advance care planning, advance directives, and medical ethical aspects have to be considered carefully before the initiation of therapy. Furthermore, the determination of no further escalation of therapy with ASDs, or their discontinuation in the context of terminal care, has to be considered and re-evaluated on a regular basis depending on the individual's clinical course and the ACP or directives.

\section{Compliance with Ethical Standards}

Funding Open Access funding enabled and organized by Projekt DEAL. The authors were supported via the Center for Personalized and Translational Epilepsy Research with a LOEWE grant from the State of Hessen.

Conflict of interest Felix Rosenow reports grants and personal fees from UCB Pharma, Arvelle Therapeutics, and Desitin Arzneimittel; personal fees from Eisai, GW Pharmaceuticals, Novartis, Medtronic, Cerbomed, Sandoz, BayerVital, and Shire; and grants from the European Union, Deutsche Forschungsgemeinschaft, the LOEWEProgramm of the state of Hesse, and the Detlev-Wrobel-Fonds for Epilepsy Research. Adam Strzelczyk reports personal fees and grants from Arvelle Therapeutics, Desitin Arzneimittel, Eisai, GW Pharmaceuticals, LivaNova, Marinus Pharmaceuticals, Medtronic, Sage Therapeutics, UCB Pharma, and Zogenix. Laurent M. Willems, Sebastian Bauer, Martin Voss, and Kolja Jahnke report no conflicts of interest. The authors have no other relevant affiliations or financial involvement with any organization or entity with a financial interest in or financial conflict with the subject matter or materials discussed in this manuscript apart from those disclosed.

Consent for publication All authors approved the final manuscript for submission.

Author contributions LMW developed the idea for this review. AS and LMW drafted the concept and performed the literature search and data analysis. LMW and AS wrote the manuscript, and SB, KJ, MV and FR critically revised the work. All authors contributed to the final manuscript.

Open Access This article is licensed under a Creative Commons Attribution 4.0 International License, which permits use, sharing, adaptation, distribution and reproduction in any medium or format, as long as you give appropriate credit to the original author(s) and the source, provide a link to the Creative Commons licence, and indicate if changes were made. The images or other third party material in this article are included in the article's Creative Commons licence, unless indicated otherwise in a credit line to the material. If material is not included in the article's Creative Commons licence and your intended use is not 
permitted by statutory regulation or exceeds the permitted use, you will need to obtain permission directly from the copyright holder. To view a copy of this licence, visit http://creativecommons.org/licenses/by/4.0/.

\section{References}

1. Trinka E, Kalviainen R. 25 years of advances in the definition, classification and treatment of status epilepticus. Seizure. 2017;44:65-73.

2. Shorvon S, Ferlisi M. The treatment of super-refractory status epilepticus: a critical review of available therapies and a clinical treatment protocol. Brain. 2011;134(Pt 10):2802-18.

3. Trinka E, Cock H, Hesdorffer D, Rossetti AO, Scheffer IE, Shinnar S, et al. A definition and classification of status epilepticus-report of the ILAE Task Force on Classification of Status Epilepticus. Epilepsia. 2015;56(10):1515-23.

4. Kapur J, Elm J, Chamberlain JM, Barsan W, Cloyd J, Lowenstein $\mathrm{D}$, et al. Randomized trial of three anticonvulsant medications for status epilepticus. N Engl J Med. 2019;381(22):2103-13.

5. Rosenow F. AWMF-Leitlinie: Status epilepticus im Erwachsenenalter; Deutsche Gesellschaft für Neurologie (DGN). In: Diener H, Weimar C, editors. Leitlinien für Diagnostik und Therapie in der Neurologie, Kapitel Anfälle und Bewusstseinsstörungen. Stuttgart: Thieme Verlag; 2017.

6. Meierkord H, Boon P, Engelsen B, Gocke K, Shorvon S, Tinuper $\mathrm{P}$, et al. EFNS guideline on the management of status epilepticus in adults. Eur J Neurol. 2010;17(3):348-55.

7. Glauser T, Shinnar S, Gloss D, Alldredge B, Arya R, Bainbridge $\mathrm{J}$, et al. Evidence-based guideline: treatment of convulsive status epilepticus in children and adults: report of the Guideline Committee of the American Epilepsy Society. Epilepsy Curr. 2016;16(1):48-61.

8. Brophy GM, Bell R, Claassen J, Alldredge B, Bleck TP, Glauser $\mathrm{T}$, et al. Guidelines for the evaluation and management of status epilepticus. Neurocrit Care. 2012;17(1):3-23.

9. Gomes D, Pimentel J, Bentes C, Aguiar de Sousa D, Antunes AP, Alvarez A, et al. Consensus protocol for the treatment of super-refractory status epilepticus. Acta Med Port. 2018;31(10):598-605.

10. Holtkamp M. Pharmacotherapy for refractory and super-refractory status epilepticus in adults. Drugs. 2018;78(3):307-26.

11. Schubert-Bast S, Zöllner JP, Ansorge S, Hapfelmeier J, Bonthapally V, Eldar-Lissai A, et al. Burden and epidemiology of status epilepticus in infants, children, and adolescents: a population-based study on German health insurance data. Epilepsia. 2019;60(5):911-20.

12. Strzelczyk A, Ansorge S, Hapfelmeier J, Bonthapally V, Erder MH, Rosenow F. Costs, length of stay, and mortality of superrefractory status epilepticus: a population-based study from Germany. Epilepsia. 2017;58(9):1533-41.

13. Kortland LM, Alfter A, Bähr O, Carl B, Dodel R, Freiman TM, et al. Costs and cost-driving factors for acute treatment of adults with status epilepticus: a multicenter cohort study from Germany. Epilepsia. 2016;57(12):2056-66.

14. Kortland LM, Knake S, von Podewils F, Rosenow F, Strzelczyk A. Socioeconomic outcome and quality of life in adults after status epilepticus: a multicenter, longitudinal, matched case-control analysis from Germany. Front Neurol. 2017;8:507.

15. Etkind SN, Bone AE, Gomes B, Lovell N, Evans CJ, Higginson IJ, et al. How many people will need palliative care in 2040? Past trends, future projections and implications for services. BMC Med. 2017;15(1):102.
16. Lupu D, Quigley L, Mehfoud N, Salsberg ES. The growing demand for hospice and palliative medicine physicians: will the supply keep up? J Pain Symptom Manag. 2018;55(4):1216-23.

17. McCormick AJ. Self-determination, the right to die, and culture: a literature review. Soc Work. 2011;56(2):119-28.

18. Gronheit W, Popkirov S, Wehner T, Schlegel U, Wellmer J. Practical management of epileptic seizures and status epilepticus in adult palliative care patients. Front Neurol. 2018;9:595.

19. Kalviainen R, Reinikainen M. Management of prolonged epileptic seizures and status epilepticus in palliative care patients. Epilepsy Behav. 2019;101(Pt B):106288.

20. Landefeld J, Incze MA. Advanced care planning — what should i know? JAMA Intern Med. 2019. https://doi.org/10.1001/jamai nternmed.2019.0005.

21. Zubek L. Options for the improvement of communication and self-determination in end-of-life decisions in intensive care units [in Hungarian]. Orv Hetil. 2016;157(17):669-74.

22. Mori H, Takahashi K, Mizutani T. Interaction between valproic acid and carbapenem antibiotics. Drug Metab Rev. 2007;39(4):647-57.

23. Moher D, Altman DG, Liberati A, Tetzlaff J. PRISMA statement. Epidemiology. 2011;22(1):128.

24. Liberati A, Altman DG, Tetzlaff J, Mulrow C, Gotzsche PC, Ioannidis JP, et al. The PRISMA statement for reporting systematic reviews and meta-analyses of studies that evaluate health care interventions: explanation and elaboration. J Clin Epidemiol. 2009;62(10):e1-34.

25. Schiavo JH. PROSPERO: an international register of systematic review protocols. Med Ref Serv Q. 2019;38(2):171-80.

26. Zeiler FA, Matuszczak M, Teitelbaum J, Gillman LM, Kazina CJ. Magnesium sulfate for non-eclamptic status epilepticus. Seizure. 2015;32:100-8.

27. Appavu B, Vanatta L, Condie J, Kerrigan JF, Jarrar R. Ketogenic diet treatment for pediatric super-refractory status epilepticus. Seizure. 2016;41:62-5.

28. Bauer S, Willems LM, Paule E, Petschow C, Zollner JP, Rosenow $\mathrm{F}$, et al. The efficacy of lacosamide as monotherapy and adjunctive therapy in focal epilepsy and its use in status epilepticus: clinical trial evidence and experience. Ther Adv Neurol Disord. 2017;10(2):103-26.

29. Brigo F, Bragazzi NL, Igwe SC, Nardone R, Trinka E. Topiramate in the treatment of generalized convulsive status epilepticus in adults: a systematic review with individual patient data analysis. Drugs. 2017;77(1):67-74.

30. Brigo F, Lattanzi S, Rohracher A, Russo E, Meletti S, Grillo E, et al. Perampanel in the treatment of status epilepticus: a systematic review of the literature. Epilepsy Behav. 2018;86:179-86.

31. Cervenka MC, Hartman AL, Venkatesan A, Geocadin RG, Kossoff EH. The ketogenic diet for medically and surgically refractory status epilepticus in the neurocritical care unit. Neurocrit Care. 2011;15(3):519-24.

32. Farrokh S, Bon J, Erdman M, Tesoro E. Use of newer anticonvulsants for the treatment of status epilepticus. Pharmacotherapy. 2019;39(3):297-316.

33. Fernandez EM, Franck AJ. Lacosamide for the treatment of refractory status epilepticus. Ann Pharmacother. 2011;45(11):1445-9.

34. Strzelczyk A, Klein KM, Willems LM, Rosenow F, Bauer S. Brivaracetam in the treatment of focal and idiopathic generalized epilepsies and of status epilepticus. Expert Rev Clin Pharmacol. 2016;9(5):637-45.

35. Strzelczyk A, Willems LM, Willig S, Rosenow F, Bauer S. Perampanel in the treatment of focal and idiopathic generalized epilepsies and of status epilepticus. Expert Rev Clin Pharmacol. 2015;8(6):733-40. 
36. Strzelczyk A, Zöllner JP, Willems LM, Jost J, Paule E, SchubertBast $\mathrm{S}$, et al. Lacosamide in status epilepticus: systematic review of current evidence. Epilepsia. 2017;58(6):933-50.

37. Willems LM, Bauer S, Rosenow F, Strzelczyk A. Recent advances in the pharmacotherapy of epilepsy: brivaracetam and perampanel as broad-spectrum antiseizure drugs for the treatment of epilepsies and status epilepticus. Expert Opin Pharmacother. 2019;20(14):1755-65.

38. Williams TJ, Cervenka MC. The role for ketogenic diets in epilepsy and status epilepticus in adults. Clin Neurophysiol Pract. 2017;2:154-60.

39. Zeiler FA, Matuszczak M, Teitelbaum J, Kazina CJ, Gillman LM. Intravenous immunoglobulins for refractory status epilepticus, part I: a scoping systematic review of the adult literature. Seizure. 2017;45:172-80.

40. Zeiler FA, Matuszczak M, Teitelbaum J, Kazina CJ, Gillman LM. Plasmapheresis for refractory status epilepticus. Part II: a scoping systematic review of the pediatric literature. Seizure. 2016;43:61-8.

41. Zeiler FA, Matuszczak M, Teitelbaum J, Kazina CJ, Gillman LM. Plasmapheresis for refractory status epilepticus, part I: a scoping systematic review of the adult literature. Seizure. 2016;43:14-22.

42. Oxford Centre for Evidence-based Medicine. Levels of Evidence (March 2009). Oxford: Centre for Evidence-Based Medicine; 2009.

43. Balshem H, Helfand M, Schunemann HJ, Oxman AD, Kunz R, Brozek J, et al. GRADE guidelines: 3. Rating the quality of evidence. J Clin Epidemiol. 2011;64(4):401-6.

44. Fisher RS, Cross JH, French JA, Higurashi N, Hirsch E, Jansen $\mathrm{FE}$, et al. Operational classification of seizure types by the International League Against Epilepsy: position paper of the ILAE Commission for Classification and Terminology. Epilepsia. 2017;58(4):522-30.

45. Scheffer IE, Berkovic S, Capovilla G, Connolly MB, French J, Guilhoto L, et al. ILAE classification of the epilepsies: position paper of the ILAE Commission for Classification and Terminology. Epilepsia. 2017;58(4):512-21.

46. Salins N, Gursahani R, Mathur R, Iyer S, Macaden S, Simha $\mathrm{N}$, et al. Definition of terms used in limitation of treatment and providing palliative care at the end of life: the Indian Council of Medical Research Commission Report. Indian J Crit Care Med. 2018;22(4):249-62.

47. Willems LM, Bertsche A, Bosebeck F, Hornemann F, Immisch I, Klein KM, et al. Efficacy, retention, and tolerability of brivaracetam in patients with epileptic encephalopathies: a multicenter cohort study from Germany. Front Neurol. 2018;9:569.

48. Schubert-Bast S, Willems LM, Kurlemann G, Knake S, MüllerSchlüter K, Rosenow F, Strzelczyk A. Postmarketing experience with brivaracetam in the treatment of focal epilepsy in children and adolescents. Epilepsy Behav. 2018;89:89-93.

49. Strzelczyk A, Steinig I, Willems LM, Reif PS, Senft C, Voss M, et al. Treatment of refractory and super-refractory status epilepticus with brivaracetam: a cohort study from two German university hospitals. Epilepsy Behav. 2017;70:177-81.

50. Santamarina E, Parejo Carbonell B, Sala J, Gutierrez-Viedma A, Miro J, Asensio M, et al. Use of intravenous brivaracetam in status epilepticus: a multicenter registry. Epilepsia. 2019;60(8):1593-601.

51. Kalss G, Rohracher A, Leitinger M, Pilz G, Novak HF, Neuray C, et al. Intravenous brivaracetam in status epilepticus: a retrospective single-center study. Epilepsia. 2018;59(Suppl 2):228-33.

52. Strzelczyk A, Kay L, Bauer S, Immisch I, Klein KM, Knake S, et al. Use of brivaracetam in genetic generalized epilepsies and for acute, intravenous treatment of absence status epilepticus. Epilepsia. 2018;59(8):1549-56.
53. Aicua-Rapun I, Andre P, Rossetti AO, Decosterd LA, Buclin T, Novy J. Intravenous brivaracetam in status epilepticus: correlation between loading dose, plasma levels and clinical response. Epilepsy Res. 2019;149:88-91.

54. Willems LM, Zollner JP, Paule E, Schubert-Bast S, Rosenow F, Strzelczyk A. Eslicarbazepine acetate in epilepsies with focal and secondary generalised seizures: systematic review of current evidence. Expert Rev Clin Pharmacol. 2018;11(3):309-24.

55. Kellinghaus C, Berning S, Immisch I, Larch J, Rosenow F, Rossetti AO, et al. Intravenous lacosamide for treatment of status epilepticus. Acta Neurol Scand. 2011;123(2):137-41.

56. Albers JM, Moddel G, Dittrich R, Steidl C, Suntrup S, Ringelstein EB, et al. Intravenous lacosamide-an effective add-on treatment of refractory status epilepticus. Seizure. 2011;20(5):428-30.

57. Goodwin H, Hinson HE, Shermock KM, Karanjia N, Lewin JJ 3rd. The use of lacosamide in refractory status epilepticus. Neurocrit Care. 2011;14(3):348-53.

58. Höfler J, Unterberger I, Dobesberger J, Kuchukhidze G, Walser G, Trinka E. Intravenous lacosamide in status epilepticus and seizure clusters. Epilepsia. 2011;52(10):e148-52.

59. Koubeissi MZ, Mayor CL, Estephan B, Rashid S, Azar NJ. Efficacy and safety of intravenous lacosamide in refractory nonconvulsive status epilepticus. Acta Neurol Scand. 2011;123(2):142-6.

60. Rantsch K, Walter U, Wittstock M, Benecke R, Rosche J. Efficacy of intravenous lacosamide in refractory nonconvulsive status epilepticus and simple partial status epilepticus. Seizure. 2011;20(7):529-32.

61. Jain V, Harvey AS. Treatment of refractory tonic status epilepticus with intravenous lacosamide. Epilepsia. 2012;53(4):761-2.

62. Cherry S, Judd L, Muniz JC, Elzawahry H, LaRoche S. Safety and efficacy of lacosamide in the intensive care unit. Neurocrit Care. 2012;16(2):294-8.

63. Mnatsakanyan L, Chung JM, Tsimerinov EI, Eliashiv DS. Intravenous lacosamide in refractory nonconvulsive status epilepticus. Seizure. 2012;21(3):198-201.

64. Belcastro V, Vidale S, Pierguidi L, Sironi L, Tancredi L, Striano $\mathrm{P}$, et al. Intravenous lacosamide as treatment option in post-stroke non convulsive status epilepticus in the elderly: a proof-of-concept, observational study. Seizure. 2013;22(10):905-7.

65. Miró J, Toledo M, Santamarina E, Ricciardi AC, Villanueva V, Pato A, et al. Efficacy of intravenous lacosamide as an add-on treatment in refractory status epilepticus: a multicentric prospective study. Seizure. 2013;22(1):77-9.

66. Santamarina E, Toledo M, Sueiras M, Raspall M, Ailouti N, Lainez E, et al. Usefulness of intravenous lacosamide in status epilepticus. J Neurol. 2013;260(12):3122-8.

67. Sutter R, Marsch S, Rüegg S. Safety and efficacy of intravenous lacosamide for adjunctive treatment of refractory status epilepticus: a comparative cohort study. CNS Drugs. 2013;27(4):321-9.

68. Legros B, Depondt C, Levy-Nogueira M, Ligot N, Mavroudakis $\mathrm{N}$, Naeije G, et al. Intravenous lacosamide in refractory seizure clusters and status epilepticus: comparison of 200 and $400 \mathrm{mg}$ loading doses. Neurocrit Care. 2014;20(3):484-8.

69. Kellinghaus C, Berning S, Stogbauer F. Intravenous lacosamide or phenytoin for treatment of refractory status epilepticus. Acta Neurol Scand. 2014;129(5):294-9.

70. Garcés M, Villanueva V, Mauri JA, Suller A, Garcia C, Lopez Gonzalez FJ, et al. Factors influencing response to intravenous lacosamide in emergency situations: LACO-IV study. Epilepsy Behav. 2014;36:144-52.

71. Grosso S, Zamponi N, Bartocci A, Cesaroni E, Cappanera S, Di Bartolo R, et al. Lacosamide in children with refractory status epilepticus. A multicenter Italian experience. Eur J Paediatr Neurol. 2014;18(5):604-8. 
72. Poddar K, Sharma R, Ng YT. Intravenous lacosamide in pediatric status epilepticus: an open-label efficacy and safety study. Pediatr Neurol. 2016;61:83-6.

73. Moreno Morales EY, Fernandez Peleteiro M, Bondy Pena EC, Dominguez Lorenzo JM, Pardellas Santiago E, Fernandez A. Observational study of intravenous lacosamide in patients with convulsive versus non-convulsive status epilepticus. Clin Drug Investig. 2015;35(7):463-9.

74. d'Orsi G, Pascarella MG, Martino T, Specchio LM. Lacosamide in absence status epilepticus: effective or ineffective? Seizure. 2015;25:32.

75. Lang N, Lange M, Schmitt FC, Bos M, Weber Y, Evers S, et al. Intravenous lacosamide in clinical practice-results from an independent registry. Seizure. 2016;39:5-9.

76. Santamarina E, Gonzalez-Cuevas M, Toledo M, Jimenez M, Becerra JL, Quilez A, et al. Intravenous lacosamide (LCM) in status epilepticus (SE): weight-adjusted dose and efficacy. Epilepsy Behav. 2018;84:93-8.

77. Reif PS, Manner A, Willems LM, Kay L, Zöllner JP, Klein KM, et al. Intravenous lacosamide for treatment of absence status epilepticus in genetic generalized epilepsy: a case report and review of literature. Acta Neurol Scand. 2018;138(3):259-62.

78. Ngampoopun M, Suwanpakdee P, Jaisupa N, Nabangchang C. Effectiveness and adverse effect of intravenous lacosamide in nonconvulsive status epilepticus and acute repetitive seizures in children. Neurol Res Int. 2018;2018:8432859.

79. Perrenoud M, Andre P, Alvarez V, Stahli C, Decosterd LA, Rossetti AO, et al. Intravenous lacosamide in status epilepticus: correlation between loading dose, serum levels, and clinical response. Epilepsy Res. 2017;135:38-42.

80. Newey CR, Le NM, Ahrens C, Sahota P, Hantus S. The safety and effectiveness of intravenous lacosamide for refractory status epilepticus in the critically ill. Neurocrit Care. 2017;26(2):273-9.

81. Toledo M, Molins A, Quintana M, Santamarina E, MartinezRicarte F, Martinez-Saez E, et al. Outcome of cancer-related seizures in patients treated with lacosamide. Acta Neurol Scand. 2018;137(1):67-75.

82. Yasam VR, Jakki SL, Senthil V, Eswaramoorthy M, Shanmuganathan S, Arjunan K, et al. A pharmacological overview of lamotrigine for the treatment of epilepsy. Expert Rev Clin Pharmacol. 2016;9(12):1533-46.

83. Pisani F, Gallitto G, Di Perri R. Could lamotrigine be useful in status epilepticus? A case report. J Neurol Neurosurg Psychiatry. 1991;54(9):845-6.

84. Bialer M, Soares-Da-Silva P. Pharmacokinetics and drug interactions of eslicarbazepine acetate. Epilepsia. 2012;53(6):935-46.

85. Kellinghaus C, Berning S, Stogbauer F. Use of oxcarbazepine for treatment of refractory status epilepticus. Seizure. 2014;23(2):151-4.

86. Chen JW, Wasterlain CG. Status epilepticus: pathophysiology and management in adults. Lancet Neurol. 2006;5(3):246-56.

87. Strzelczyk A, Knake S, Kälviäinen R, Santamarina E, Toledo M, Willig S, et al. Perampanel for treatment of status epilepticus in Austria, Finland, Germany, and Spain. Acta Neurol Scand. 2019;139(4):369-76.

88. Redecker J, Wittstock M, Benecke R, Rösche J. Efficacy of perampanel in refractory nonconvulsive status epilepticus and simple partial status epilepticus. Epilepsy Behav. 2015;45:176-9.

89. Rohracher A, Höfler J, Kalss G, Leitinger M, Kuchukhidze G, Deak I, et al. Perampanel in patients with refractory and superrefractory status epilepticus in a neurological intensive care unit. Epilepsy Behav. 2015;49:354-8.

90. Beretta S, Padovano G, Stabile A, Coppo A, Bogliun G, Avalli L, et al. Efficacy and safety of perampanel oral loading in postanoxic super-refractory status epilepticus: a pilot study. Epilepsia. 2018;59(Suppl 2):243-8.
91. Rohracher A, Kalss G, Neuray C, Hofler J, Dobesberger J, Kuchukhidze G, et al. Perampanel in patients with refractory and super-refractory status epilepticus in a neurological intensive care unit: a single-center audit of 30 patients. Epilepsia. 2018;59(Suppl 2):234-42.

92. Newey CR, Mullaguri N, Hantus S, Punia V, George P. Superrefractory status epilepticus treated with high dose perampanel: case series and review of the literature. Case Rep Crit Care. 2019;2019:3218231.

93. Ho CJ, Lin CH, Lu YT, Shih FY, Hsu CW, Tsai WC, et al. Perampanel treatment for refractory status epilepticus in a neurological intensive care unit. Neurocrit Care. 2019;31(1):24-9.

94. Rahbani A, Adwane G, Jomaa N. Oral perampanel for the treatment of super-refractory status epilepticus. Case Rep Neurol Med. 2019;2019:8537815.

95. Santamarina E, Alpuente A, Maisterra O, Sueiras M, Sarria S, Guzman L, et al. Perampanel: a therapeutic alternative in refractory status epilepticus associated with MELAS syndrome. Epilepsy Behav Case Rep. 2019;11:92-5.

96. Strzelczyk A, Kortland LM, Knake S, Rosenow F. Stiripentol for the treatment of super-refractory status epilepticus. Acta Neurol Scand. 2015;132(6):435-9.

97. Fisher JL. The anti-convulsant stiripentol acts directly on the GABA(A) receptor as a positive allosteric modulator. Neuropharmacology. 2009;56(1):190-7.

98. Uchida Y, Kato D, Toyoda T, Oomura M, Ueki Y, Ohkita K, et al. Combination of ketogenic diet and stiripentol for superrefractory status epilepticus: a case report. J Neurol Sci. 2017;15(373):35-7.

99. Uchida Y, Terada K, Madokoro Y, Fujioka T, Mizuno M, Toyoda T, et al. Stiripentol for the treatment of super-refractory status epilepticus with cross-sensitivity. Acta Neurol Scand. 2018;137(4):432-7.

100. Kahriman M, Minecan D, Kutluay E, Selwa L, Beydoun A. Efficacy of topiramate in children with refractory status epilepticus. Epilepsia. 2003;44(10):1353-6.

101. Bensalem MK, Fakhoury TA. Topiramate and status epilepticus: report of three cases. Epilepsy Behav. 2003;4(6):757-60.

102. Blumkin L, Lerman-Sagie T, Houri T, Gilad E, Nissenkorn A, Ginsberg M, et al. Pediatric refractory partial status epilepticus responsive to topiramate. J Child Neurol. 2005;20(3):239-41.

103. Perry MS, Holt PJ, Sladky JT. Topiramate loading for refractory status epilepticus in children. Epilepsia. 2006;47(6):1070-1.

104. Soler B, Godoy J, Mellado TP. Treatment of refractory status epilepticus with topiramate. Report of three cases. Rev Med Chil. 2009;137(7):936-9.

105. Akyildiz BN, Kumandas S. Treatment of pediatric refractory status epilepticus with topiramate. Childs Nerv Syst. 2011;27(9):1425-30.

106. Kim W, Kwon SY, Cho AH, Lim SC, Kim YI, Shon YM. Effectiveness of topiramate in medically complicated patients with status epilepticus or acute refractory seizures. J Epilepsy Res. 2011;1(2):52-6.

107. Synowiec AS, Yandora KA, Yenugadhati V, Valeriano JP, Schramke CJ, Kelly KM. The efficacy of topiramate in adult refractory status epilepticus: experience of a tertiary care center. Epilepsy Res. 2012;98(2-3):232-7.

108. Bragatti JA, Torres CM, Netto CB, Vedolin L, Garzon E, Rieder $\mathrm{CR}$, et al. Topiramate is effective for status epilepticus and seizure control in neuraminidase deficiency. Arq Neuropsiquiatr. 2011;69(3):565-6.

109. Hottinger A, Sutter R, Marsch S, Ruegg S. Topiramate as an adjunctive treatment in patients with refractory status epilepticus: an observational cohort study. CNS Drugs. 2012;26(9):761-72. 
110. Stojanova V, Rossetti AO. Oral topiramate as an add-on treatment for refractory status epilepticus. Acta Neurol Scand. 2012;125(2):e7-e11.

111. Shelton CM, Alford EL, Storgion S, Wheless J, Phelps SJ. Enteral topiramate in a pediatric patient with refractory status epilepticus: a case report and review of the literature. J Pediatr Pharmacol Ther. 2014;19(4):317-24.

112. Asadi-Pooya AA, Jahromi MJ, Izadi S, Emami Y. Treatment of refractory generalized convulsive status epilepticus with enteral topiramate in resource limited settings. Seizure. 2015;24:114-7.

113. Madzar D, Kuramatsu JB, Gerner ST, Huttner HB. Assessing the value of topiramate in refractory status epilepticus. Seizure. 2016;38:7-10.

114. Fechner A, Hubert K, Jahnke K, Knake S, Konczalla J, Menzler $\mathrm{K}$, et al. Treatment of refractory and superrefractory status epilepticus with topiramate: a cohort study of 106 patients and a review of the literature. Epilepsia. 2019;60(12):2448-58.

115. Mirza N, Marson AG, Pirmohamed M. Effect of topiramate on acid-base balance: extent, mechanism and effects. Br J Clin Pharmacol. 2009;68(5):655-61.

116. Hubert K, Knake S, Bauer S, Voss M, Rosenow F, Strzelczyk A. Treatment of status epilepticus with zonisamide: a multicenter cohort study of 34 patients and review of literature. Epilepsy Behav. 2020;14(109):107139.

117. Baxter P, Clarke A, Cross H, Harding B, Hicks E, Livingston J, et al. Idiopathic catastrophic epileptic encephalopathy presenting with acute onset intractable status. Seizure. 2003;12(6):379-87.

118. Berkeley J, Foreman P, Foroughi A, Tirol F. Successful treatment of febrile illness related epilepsy syndrom (FIRES) and new onset refractory status epilepticus (NORSE) with plasma exchange. Epilepsy Curr. 2015;15:275.

119. Broomall E, Natale JE, Grimason M, Goldstein J, Smith CM, Chang C, et al. Pediatric super-refractory status epilepticus treated with allopregnanolone. Ann Neurol. 2014;76(6):911-5.

120. Dionisio S, Brown H, Lander C, Airey C, Lehn A, Nooruddin H, et al. Immunoglobulin-responsive refractory epilepsy-3 cases with a similar EEG pattern. Seizure. 2013;22(5):403-8.

121. Fisher RS, Kaplan PW, Krumholz A, Lesser RP, Rosen SA, Wolff MR. Failure of high-dose intravenous magnesium sulfate to control myoclonic status epilepticus. Clin Neuropharmacol. 1988;11(6):537-44.

122. Gedik AH, Demirkol D, Tatli B, Bayraktar S, Alkan A, Karabocuoglu M, et al. Therapeutic plasma exchange for malignant refractory status epilepticus: a case report. Pediatr Neurol. 2014;50(4):407-10.

123. Nandakumar A, Andrezejowski J, Turnbull D. Case report: new onset drug resistant status epilepticus (NODRSE). J Neurosurg Anesthesiol. 2008;20:218

124. Neligan A, Oomeer S, Ziso B, Chrisofi G, Turner B. A case of prolonged status epilepticus with a good outcome: the importance of etiology in determining prognosis. Epilepsia. 2011;52:83.

125. Pandey M, Gupta A, Baduni N, Vijfdar H, Sinha S, Jain A. Refractory status epilepticus-magnesium as rescue therapy. Anaesth Intensive Care. 2010;38(5):962.

126. Robakis TK, Hirsch LJ. Literature review, case report, and expert discussion of prolonged refractory status epilepticus. Neurocrit Care. 2006;4(1):35-46.

127. Sadeh M, Blatt I, Martonovits G, Karni A, Goldhammer Y. Treatment of porphyric convulsions with magnesium sulfate. Epilepsia. 1991;32(5):712-5.

128. Sahin M, Menache CC, Holmes GL, Riviello JJ. Outcome of severe refractory status epilepticus in children. Epilepsia. 2001;42(11):1461-7.
129. Savard M, Dupre N, Turgeon AF, Desbiens R, Langevin S, Brunet D. POLG mitochondrial disorder heralded by propofol infusion syndrome: a case report. Neurol Sci. 2012;39:24.

130. Shin RK, Rosenbaum AV, Frost N. A case of magnesium-responsive paraneoplastic non-convulsive status epilepticus. Ann Neurol. 2011;70:35-6.

131. Storcheim F. Status epilepticus treated by magnesium sulfate injected intravenously. JAMA. 1933;101:1313-4.

132. Strzelczyk A, Reif PS, Bauer S, Belke M, Oertel WH, Knake S, et al. Intravenous initiation and maintenance of ketogenic diet: proof of concept in super-refractory status epilepticus. Seizure. 2013;22(7):581-3.

133. Tan WW, Chan DWS, Lee JH, Thomas T, Menon AP, Chan YH. Use of magnesium sulfate infusion for the management of febrile illness-related epilepsy syndrome: a case series. Child Neurol Open. 2015;2(1):2329048X14550067.

134. Valle-Morales L, Cortes-Cros E, Santana A, Barber M, Figueras T, Garcia-Hernandez JA. Epileptic status refractory to conventional treatment caused by vitamin B6 deficiency. J Perinatol. 2009;29(3):252-3.

135. Visser NA, Braun KP, Leijten FS, van Nieuwenhuizen O, Wokke $\mathrm{JH}$, van den Bergh WM. Magnesium treatment for patients with refractory status epilepticus due to POLG1-mutations. J Neurol. 2011;258(2):218-22.

136. Madisia N, Bergkeley JL. Successful treatment of prolonged refravtory status epilepticus with plasma exchange and rituximab. Neurocrit Care. 2014;21:280.

137. Zaatreh MM. Levetiracetam in porphyric status epilepticus: a case report. Clin Neuropharmacol. 2005;28(5):243-4.

138. Hatch DM, Atito-Narh E, Herschmiller EJ, Olufolabi AJ, Owen MD. Refractory status epilepticus after inadvertent intrathecal injection of tranexamic acid treated by magnesium sulfate. Int $\mathrm{J}$ Obstet Anesth. 2016;26:71-5.

139. Sahin M, Riviello JJ Jr. Prolonged treatment of refractory status epilepticus in a child. J Child Neurol. 2001;16(2):147-50.

140. Bodenant M, Moreau C, Sejourne C, Auvin S, Delval A, Cuisset JM, et al. Interest of the ketogenic diet in a refractory status epilepticus in adults [in French]. Rev Neurol (Paris). 2008;164(2):194-9.

141. Wusthoff CJ, Kranick SM, Morley JF, Christina Bergqvist AG. The ketogenic diet in treatment of two adults with prolonged nonconvulsive status epilepticus. Epilepsia. 2010;51(6):1083-5.

142. Nabbout R, Mazzuca M, Hubert P, Peudennier S, Allaire C, Flurin $\mathrm{V}$, et al. Efficacy of ketogenic diet in severe refractory status epilepticus initiating fever induced refractory epileptic encephalopathy in school age children (FIRES). Epilepsia. 2010;51(10):2033-7.

143. Kramer U, Chi CS, Lin KL, Specchio N, Sahin M, Olson H, et al. Febrile infection-related epilepsy syndrome (FIRES): does duration of anesthesia affect outcome? Epilepsia. 2011;52(Suppl 8):28-30.

144. Nam SH, Lee BL, Lee CG, Yu HJ, Joo EY, Lee J, et al. The role of ketogenic diet in the treatment of refractory status epilepticus. Epilepsia. 2011;52(11):e181-e184184.

145. Martikainen MH, Paivarinta M, Jaaskelainen S, Majamaa K. Successful treatment of POLG-related mitochondrial epilepsy with antiepileptic drugs and low glycaemic index diet. Epileptic Disord. 2012;14(4):438-41.

146. Vaccarezza M, Silva W, Maxit C, Agosta G. Super-refractory status epilepticus: treatment with ketogenic diet in pediatrics. Rev Neurol. 2012;55(1):20-5.

147. Sort R, Born AP, Pedersen KN, Fonsmark L, Uldall P. Ketogenic diet in 3 cases of childhood refractory status epilepticus. Eur J Paediatr Neurol. 2013;17(6):531-6.

148. Caraballo RH, Flesler S, Armeno M, Fortini S, Agustinho A, Mestre G, et al. Ketogenic diet in pediatric patients 
with refractory focal status epilepticus. Epilepsy Res. 2014;108(10):1912-6.

149. Cobo NH, Sankar R, Murata KK, Sewak SL, Kezele MA, Matsumoto JH. The ketogenic diet as broad-spectrum treatment for super-refractory pediatric status epilepticus: challenges in implementation in the pediatric and neonatal intensive care units. $\mathbf{J}$ Child Neurol. 2015;30(2):259-66.

150. Thakur KT, Probasco JC, Hocker SE, Roehl K, Henry B, Kossoff EH, et al. Ketogenic diet for adults in super-refractory status epilepticus. Neurology. 2014;82(8):665-70.

151. Amer S, Shah P, Kommineni V. Refractory status epilepticus from NMDA receptor encephalitis successfully treated with an adjunctive ketogenic diet. Ann Indian Acad Neurol. 2015;18(2):256-7.

152. Caraballo RH, Valenzuela GR, Armeno M, Fortini S, Mestre G, Cresta A. The ketogenic diet in two paediatric patients with refractory myoclonic status epilepticus. Epileptic Disord. 2015;17(4):491-5.

153. Cash C. Use of the ketogenic diet in an adult with myoclonic status epilepticus. Clin Nutr ESPEN. 2015;10(5):e199-e200.

154. Fung EL, Chang SK, Yam KK, Yau PY. Ketogenic diet as a therapeutic option in super-refractory status epilepticus. Pediatr Neonatol. 2015;56(6):429-31.

155. Lin JJ, Lin KL, Chan OW, Hsia SH, Wang HS, Group CS. Intravenous ketogenic diet therapy for treatment of the acute stage of super-refractory status epilepticus in a pediatric patient. Pediatr Neurol. 2015;52(4):442-5.

156. Caraballo R, Darra F, Reyes G, Armeno M, Cresta A, Mestre $\mathrm{G}$, et al. The ketogenic diet in patients with myoclonic status in non-progressive encephalopathy. Seizure. 2017;51:1-5.

157. Cervenka MC, Hocker S, Koenig M, Bar B, Henry-Barron $\mathrm{B}$, Kossoff EH, et al. Phase I/II multicenter ketogenic diet study for adult superrefractory status epilepticus. Neurology. 2017;88(10):938-43.

158. Smith G, Press CA. Ketogenic diet in super-refractory status epilepticus. Pediatr Neurol Briefs. 2017;31(3):8.

159. Arya R, Peariso K, Gainza-Lein M, Harvey J, Bergin A, Brenton $\mathrm{JN}$, et al. Efficacy and safety of ketogenic diet for treatment of pediatric convulsive refractory status epilepticus. Epilepsy Res. 2018;144:1-6.

160. Blunck JR, Newman JW, Fields RK, Croom JE. Therapeutic augmentation of ketogenic diet with a sodium-glucose cotransporter 2 inhibitor in a super-refractory status epilepticus patient. Epilepsy Behav Case Rep. 2018;10:61-4.

161. Francis BA, Fillenworth J, Gorelick P, Karanec K, Tanner A. The feasibility, safety and effectiveness of a ketogenic diet for refractory status epilepticus in adults in the intensive care unit. Neurocrit Care. 2019;30(3):652-7.

162. Park EG, Lee J, Lee J. The ketogenic diet for super-refractory status epilepticus patients in intensive care units. Brain Dev. 2019;41(5):420-7.

163. Peng P, Peng J, Yin F, Deng X, Chen C, He F, et al. Ketogenic diet as a treatment for super-refractory status epilepticus in febrile infection-related epilepsy syndrome. Front Neurol. 2019;10:423

164. Prasoppokakorn T, Jirasakuldej S, Lakananurak N. Mediumchain triglyceride ketogenic diet is effective for treatment of an adult with super-refractory status epilepticus: a case report and literature review. Eur J Clin Nutr. 2019;73(12):1594-7.

165. Noviawaty I, Olaru E, Rondello C, Fitzsimmons B, Raghavan M. Clinical reasoning: ketogenic diet in adult super-refractory status epilepticus. Neurology. 2020;94(12):541-6.

166. Rosenthal ES, Claassen J, Wainwright MS, Husain AM, Vaitkevicius $\mathrm{H}$, Raines $\mathrm{S}$, et al. Brexanolone as adjunctive therapy in super-refractory status epilepticus. Ann Neurol. 2017;82(3):342-52.
167. Vaitkevicius H, Husain AM, Rosenthal ES, Rosand J, Bobb W, Reddy K, et al. First-in-man allopregnanolone use in super-refractory status epilepticus. Ann Clin Transl Neurol. 2017;4(6):411-4.

168. SageTherapeutics. A study with SAGE-547 for super-refractory status epilepticus. ClinicalTrails.gov; 2019. https://clinicaltrials. gov/ct2/show/NCT02477618.

169. Ramos AB, Cruz RA, Villemarette-Pittman NR, Olejniczak PW, Mader EC Jr. Dexamethasone as abortive treatment for refractory seizures or status epilepticus in the inpatient setting. J Investig Med High Impact Case Rep. 2019;7:2324709619848816.

170. Trinka E, Hofler J, Leitinger M, Brigo F. Pharmacotherapy for status epilepticus. Drugs. 2015;75(13):1499-521.

171. Zolkowska D, Wu CY, Rogawski MA. Intramuscular allopregnanolone and ganaxolone in a mouse model of treatment-resistant status epilepticus. Epilepsia. 2018;59(Suppl 2):220-7.

172. Rosati A, Ilvento L, Lucenteforte E, Pugi A, Crescioli G, McGreevy KS, et al. Comparative efficacy of antiepileptic drugs in children and adolescents: a network meta-analysis. Epilepsia. 2018;59(2):297-314.

173. Rosati A, De Masi S, Guerrini R. Ketamine for refractory status epilepticus: a systematic review. CNS Drugs. 2018;32(11):997-1009.

174. Dibue-Adjei M, Brigo F, Yamamoto T, Vonck K, Trinka E. Vagus nerve stimulation in refractory and super-refractory status epilepticus: a systematic review. Brain Stimul. 2019;12(5):1101-10.

175. San-Juan D, Davila-Rodriguez DO, Jimenez CR, Gonzalez MS, Carranza SM, Hernandez Mendoza JR, et al. Neuromodulation techniques for status epilepticus: a review. Brain Stimul. 2019;12(4):835-44.

176. Ferlazzo E, Sueri C, Gasparini S, Russo E, Cianci V, Ascoli $\mathrm{M}$, et al. Methodological issues associated with clinical trials in epilepsy. Expert Rev Clin Pharmacol. 2017;10(10):1103-8.

177. Perucca E, Wiebe S. Not all that glitters is gold: a guide to the critical interpretation of drug trials in epilepsy. Epilepsia Open. 2016;1(1-2):9-21.

178. Moher D, Shamseer L, Clarke M, Ghersi D, Liberati A, Petticrew $\mathrm{M}$, et al. Preferred reporting items for systematic review and meta-analysis protocols (PRISMA-P) 2015 statement. Syst Rev. $2015 ; 4(1): 1$.

179. Hopp JL, Sanchez A, Krumholz A, Hart G, Barry E. Nonconvulsive status epilepticus: value of a benzodiazepine trial for predicting outcomes. Neurologist. 2011;17(6):325-9.

180. Niquet J, Baldwin R, Norman K, Suchomelova L, Lumley L, Wasterlain CG. Simultaneous triple therapy for the treatment of status epilepticus. Neurobiol Dis. 2017;104:41-9.

181. Willems LM, Richter S, Watermann N, Bauer S, Klein KM, Reese JP, et al. Trends in resource utilization and prescription of anticonvulsants for patients with active epilepsy in Germany from 2003 to 2013 - A ten-year overview. Epilepsy Behav. 2018;83:28-35.

182. Willems LM, Hamer HM, Knake S, Rosenow F, Reese JP, Strzelczyk A. General Trends in Prices and Prescription Patterns of Anticonvulsants in Germany between 2000 and 2017: Analysis of National and Cohort-Based Data. Appl Health Econ Health Policy. 2019;17(5):707-22.

183. Rybitschka A, Semmlack S, Kaplan PW, De Marchis GM, Ruegg S, Marsch S, et al. Calorie intake during status epilepticus and outcome: a 5-year cohort study. Crit Care Med. 2019;47(8):1106-15.

184. Shorvon S. Super-refractory status epilepticus: an approach to therapy in this difficult clinical situation. Epilepsia. 2011;52(Suppl 8):53-6.

185. Arya R, Rotenberg A. Dietary, immunological, surgical, and other emerging treatments for pediatric refractory status epilepticus. Seizure. 2019;68:89-96. 
186. Bayrlee A, Ganeshalingam N, Kurczewski L, Brophy GM. Treatment of super-refractory status epilepticus. Curr Neurol Neurosci Rep. 2015;15(10):66.

187. Mattozzi S, Sabater L, Escudero D, Arino H, Armangue T, Simabukuro M, et al. Hashimoto encephalopathy in the 21 st century. Neurology. 2020;94(2):e217-e224224.

188. Kassab M, Oatel A, Shah H, Schulte A, Jani V, Burghardt T. Demographic, regional and social predictors of do not resuscitate order utilization in generalized convulsive status epilepticus (GCSE): a national perspective (P1.066). Neurology. 2016;86(16 Suppl).

189. Lunagariya A, Pateel A, Grayson J, Heilmann K. Ethical dilemma: a national perspective on utilization of do-not-resuscitate orders in status epilepticus (P1.355). Neurology. 2016;86(16 Suppl).

190. Sutter R, Meyer-Zehnder B, Baumann SM, Marsch S, Pargger H. Advance directives in the neurocritically ill: a systematic review. Crit Care Med. 2020. https://doi.org/10.1097/CCM.0000000000 004388.

191. Sutter R, De Marchis GM, Semmlack S, Fuhr P, Rüegg S, Marsch $\mathrm{S}$, et al. Anesthetics and outcome in status epilepticus: a matched two-center cohort study. CNS Drugs. 2017;31(1):65-74.

192. Marchi NA, Novy J, Faouzi M, Stahli C, Burnand B, Rossetti AO. Status epilepticus: impact of therapeutic coma on outcome. Crit Care Med. 2015;43(5):1003-9.

193. Kowalski RG, Ziai WC, Rees RN, Werner JK Jr, Kim G, Goodwin $\mathrm{H}$, et al. Third-line antiepileptic therapy and outcome in status epilepticus: the impact of vasopressor use and prolonged mechanical ventilation. Crit Care Med. 2012;40(9):2677-84.

194. Naisbitt C, Davies S. Starvation, exercise and the stress response. Anaesth Intensive Care Med. 2017;18(10):508-12.

195. Riechmann J, Willems LM, Boor R, Kieslich M, Knake S, Langner C, et al. Quality of life and correlating factors in children, adolescents with epilepsy, and their caregivers: A cross-sectional multicenter study from Germany. Seizure. 2019;69:92-8.

196. Ferlisi M, Shorvon S. The outcome of therapies in refractory and super-refractory convulsive status epilepticus and recommendations for therapy. Brain. 2012;135(Pt 8):2314-28.

197. Kantanen AM, Sairanen J, Kalviainen R. Incidence of the different stages of status epilepticus in Eastern Finland: a populationbased study. Epilepsy Behav. 2019;101(Pt B):106413.

198. Delaj L, Novy J, Ryvlin P, Marchi NA, Rossetti AO. Refractory and super-refractory status epilepticus in adults: a 9-year cohort study. Acta Neurol Scand. 2017;135(1):92-9.

199. Agan K, Midi I, Alibas H, Gonul O. Refractory status epilepticus with possible autoimmune etiology treated with plasma exchange. J Neurol Sci. 2015;357:e142-e160160.

200. Agirre-Arrizubieta Z, Moran N. Super-refractory status-epilepticus: there is always hope. Epilepsia. 2012;53.

201. Al-Ajlan FS, Althobiti A, Baz S, Al-Attas A. Autoimmune encephalopathy and drug refractory seizures with the presence of two autoantibodies specific for the neuronal cell surface. Epilepsy Behav Case Rep. 2014;2:199-202.

202. Alam H, Kassar D, Chand P, Iyadurai S. Treatment of recurrent status epilepticus secondary to Hashimoto's encephalitis by thyroidectomy. Neurocrit Care. 2013;19:S306.

203. Alam M, Deb R, Reddy K, Ratnam B, Lath R, Ranjan A. A rare case of status epilepticus-Rasmussen's encephalitis. Ind J Crit Care Med. 2014;18(Suppl.1).

204. Armas S, Miro J, Veciana M, Pedro J, Crorral L, Castaner S. Long-term immunosuppressive treatment in a patient with recurrent refractory status epilepticus. Epilepsia. 2013;54:111.

205. Barnes B, Kaplan P, Venkatesan A, Geocadin R. Recovery of consciousness in comatose patients with acute encephalitis and super refractory status epilepticus: impact of EEG sleep intrusions. Neurocrit Care. 2013;19:S316.
206. Bobb W, Kolls B, Ummat M, Husain A. Allopregnanolone to treat refractory status epilepticus. J clin Neurophysiol. 2014;31:297.

207. Brigo F, Vogrig A, Bratti A, Tavernelli V, Nardone R, Trinka E. Probable dysimmune epilepsia partialis continua manifesting as epileptic moving toes syndrome: electroclinical features of a challenging case. Epileptic Disord. 2018;20(4):301-12.

208. Buenache R, Morillo P, Mesequer M, Perez Caballaro C, Lorenzo G. Refractory epileptic encephalopathy related to mycoplasma pneumoniae infection. Epilepsia. 2012;53:544.

209. Buerger KJ, Zerr K, Salazar R. An unusual presentation of herpes simplex encephalitis with negative PCR. BMJ Case Rep. 2015;2015: bcr2015210522.

210. Calabrace J, Witherspoon B. Acute psychosis as the initial presentation of a 19yo with mediastinal teratoma. Neurocrit Care. 2013;19:321.

211. Caputo D, Iorio R, Vigevano F, Fusco L. Febrile infection-related epilepsy syndrome (FIRES) with super-refractory status epilepticus revealing autoimmune encephalitis due to GABAAR antibodies. Eur J Paediatr Neurol. 2018;22(1):182-5.

212. Caraballo RH, Reyes G, Avaria MF, Buompadre MC, Gonzalez $\mathrm{M}$, Fortini S, et al. Febrile infection-related epilepsy syndrome: a study of 12 patients. Seizure. 2013;22(7):553-9.

213. Charles S, Hainaut E, Cante V, Valette C, Levillain P, Guillet G. Dermato-neuro syndrome during scleromyxedema: efficacy of plasmapheresis and intravenous immunoglobulin [in French]. Ann Dermatol Venereol. 2014;141(8-9):523-7.

214. Chevret L, Husson B, Nguefack S, Nehlig A, Bouilleret V. Prolonged refractory status epilepticus with early and persistent restricted hippocampal signal MRI abnormality. J Neurol. 2008;255(1):112-6.

215. Chevret L, Falcon E, Durand O, Essouri S, Balu L, Bouilleret V. Plasmapheresis in malignant status epilepticus. Pediatr Crit Care Med. 2011;12:648.

216. Costas K, Tasker RC, Soul J, Lamb N, Li L, Bergin A. Pentobarbital, propylene glycol, and ketosis in refractory status epilepticus. Epilepsia. 2015;15:546.

217. Dishong M, Rutledge C, Winkler M, Prabahakara P. Refractory status epilepticus in a previously healthy child: a case report. J Invest Med. 2013;61:434-5.

218. Gall CR, Jumma O, Mohanraj R. Five cases of new onset refractory status epilepticus (NORSE) syndrome: outcomes with early immunotherapy. Seizure. 2013;22(3):217-20.

219. Ghamande S, Akiode I, HD W, Prince W, Fiocco G. Rituximab for refractory lupus cerebritis with status epilepticus. Am J Respir Crit Care Med. 2013;187:A2988.

220. Gonzalez R, Chari G, Peguero N, Ramirez M. New onset refractory status epilepticus. Epilepsy Curr. 2011;11(3):1114.

221. Hainsworth J, Shishido A, Theeler B, Carroll C, Fasano R. Treatment responsive GABA(B)-receptor limbic encephalitis presenting as a new-onset super-refractory status epilepticus (NORSE) in a Deployed U.S. Soldier. Epileptic Disord. 2014;16(4):486-93.

222. Hakimi R, Butchee M, Duvall J, Fields E, Hakimi A. Should a ketogenic died be considered earlier in the treatment course of adults with super-refractory status epilepticus? Neurocrit Care. 2014;21:227.

223. Hoang Q, Wohlt P, Rosenburg N. Treatment of super-refractory status epilepticus with perampanel in an intensive care unit. Crit Care Med. 2014;42:1250.

224. Howell KB, Katanyuwong K, Mackay MT, Bailey CA, Scheffer IE, Freeman JL, et al. Long-term follow-up of febrile infectionrelated epilepsy syndrome. Epilepsia. 2012;53(1):101-10.

225. Hribljan M, Tesovic G, Mise B, Roglic S. Febrile infectionrelated epilepsy syndrome in four Croatian boys. Epilepsia. 2013;54:58. 
226. Incecik F, Horoz O, Herguner O, Yildizdas D, Altunbasak S. Electroconvulsive therapy for refractory status epilepticus in a child: a case report. Indian Acad Neurol. 2015;18:364-5.

227. Kaneko J, Iizuka T, Uchino A, Hamada J. Immunotherapyresponsive acute encephalopathy presenting as generalize convulsive status epilepticus with fever: autoimmune epileptic syndrome? Clin Exp Neuroimmun. 2012;3:54.

228. Katsuse K, Kurihara M, Sugiyama Y, Kodama S, Takahashi M, Momose T, et al. Aphasic status epilepticus preceding tumefactive left hemisphere lesion in anti-MOG antibody associated disease. Mult Scler Relat Disord. 2019;27:91-4.

229. Khawaja A, Amara AW. Refractory status epilepticus: a report of two cases to illustrate the significance of GAD antibody. Neurology. 2014;82(10 Suppl).

230. Khawaja A, DeWolfe J, Miller D, Szaflarski J. New-onset refractory status epilepticus (NORSE) - the potneital role for immunotherapy. Epilepsy Behav. 2015;47:17-23.

231. Kirkpatrick MP, Clarke CD, Sonmezturk HH, Abou-Khalil B. Rhythmic delta activity represents a form of nonconvulsive status epilepticus in anti-NMDA receptor antibody encephalitis. Epilepsy Behav. 2011;20(2):392-4.

232. Kong SS, Chen YJ, Su IC, Lin JJ, Chou IJ, Chou ML, et al. Immunotherapy for anti-NMDA receptor encephalitis: experience from a single center in Taiwan. Pediatr Neonatol. 2019;60(4):417-22.

233. Korff C, Fluss J, Valenza N, Deglise S, Heritier-Barras A-C, Picard F. Febrile infection responsive encephalopathy of schoolage (FIRES) with unexpected favorable evolution. Epilepsia. 2009;50:84.

234. Kramer U, Chi CS, Lin KL, Specchio N, Sahin M, Olson H, et al. Febrile infection-related epilepsy syndrome (FIRES): pathogenesis, treatment, and outcome: a multicenter study on 77 children. Epilepsia. 2011;52(11):1956-65.

235. Labate A, Quattrone A, Dalmau J, Gambardella A. Anti- $N$-methyl-D-aspartate-glutamic-receptor encephalitis presenting as paroxysmal exercise-induced foot weakness. Mov Disord. 2013;28(6):820-2.

236. Le Moigno L, Ternant D, Paintaud G, Thibault G, Cloarec S, Tardieu M, et al. $N$-methyl-D-aspartate receptor antibody encephalitis: Value of immunmodulatory therapy [in French]. Arch Pediatr. 2014;21:620-3.

237. Lenoir X, Sindic C, van Pesch V, El Sankari S, de Toutchaninoff M, Denays R, et al. Anti- $N$-Methyl-D-aspartat receptor encepthalitis with favourable outcome despite prolongued status epilepticus. Neurocrit Care. 2013;18:89-92.

238. Li J, Saldivar C, Maganti R. Plasma exchange in cryptogenic new onset refractory status epilepticus. Seizure. 2013;22(1):70-3.

239. Lin JJ, Wang Y, Lan SY, Chan OW, Hsia SH, Chou ML, et al. Combination of intravenous immunoglobulin and steroid pulse therapy improves outcomes of febrile refractory status epilepticus. Epilepsy Res. 2018;142:100-5.

240. Lousa M, Sanchez-Alonso S, Rodriguez-Diaz R, Dalmau J. Status epilepticus with neuron-reactive serum antibodies: response to plasma exchange. Neurology. 2000;54:2163-5.

241. Madisi N, Berkley J. Successful treatment of prolonged status epilepticus with plasma exchange and rituximab. Neurocrit Care. 2014;21:S280.

242. Malaga A, Roncero I, Santovena-Gonzalez L, Blanco-Lago R, Rekarte S, Armangue T. PP13.2-3042: Infantile spasm in an infant with anti-NMDA receptor encephalitis secondary to HSV-1 encephalitis. Euro J Ped Neurol. 2015;19(Suppl 1):S82.

243. Mann M, Sekhon M, Javidan M. Voltage-gated potassium channel antibody associated with limbic encephalitis presenting as a rapidly progressive refractory status epilepticus: a case report and review of the literature. Neurology. 2013;80(7 Suppl).
244. Marques I, Teotónia R, Cunha C, Bento C, Sales F. Anti-NMDA receptor encephalitis presenting with total insomnia-a case report. J Neurol Sci. 2014;336(1-2):276-80.

245. Milh M, Villeneuve N, Chapon F, Gavaret M, Girard N, Mancini $\mathrm{J}$, et al. New onset refractory convulsive status epilepticus associated with serum neuropil auto-antibodies in a school aged child. Brain Dev. 2011;33(8):687-91.

246. Miras Veiga A, Moreno DC, Menendez AI, Siscart IM, Fernandez MD, Sanchez EG, et al. Effectiveness of electroconvulsive therapy for refractory status epilepticus in febrile infectionrelated epilepsy syndrome. Neuropediatrics. 2017;48(1):45-8.

247. Moeller JJ, Friedman D, Dugan P, Akman CI. Refractory status epilepticus associated with anti-SSA (anti-Ro) antibodies. Can J Neurol Sci. 2012;39(5):660-3.

248. Nakamura L. Febrile infection-related epilepsy syndrome (FIRES): a rare but very severe epileptic encephalopathy. Pediatr Radiol. 2015;45:166.

249. Noviawaty I, Valappil A, Zeft A, Lachhwani D. Super refractory status epilepticus: a case report. Epilepsy Curr. 2015;15:87.

250. Ogawa C, Natsume J, Takeuchi T, Yamamoto H, Azuma Y, Kidokoro H. First autopsy report of a patient with acute encephalitis with refractory, repetitive partial seizures. Epilepsia. 2013;54:315.

251. Pari E, Rinaldi F, Premi E, Codella M, Rao R, Paghera B, et al. A follow-up (1)(8)F-FDG brain PET study in a case of Hashimoto's encephalopathy causing drug-resistant status epilepticus treated with plasmapheresis. J Neurol. 2014;261(4):663-7.

252. Rypulak E, Borys M, Piwowarczyk P, Fijalkowska M, Potrec B, Sysiak J, et al. Successful treatment of anti-NMDA receptor encephalitis with a prompt ovarian tumour removal and prolonged course of plasmapheresis: a case report. Mol Clin Oncol. 2016;5(6):845-9.

253. Sawicka K, Cooley R, Hunter G. New onset refractory status epilepticus (NORSE) lasting 110 days resulting in a positive outcome. Neurology. 2016;86(16 Suppl).

254. Schatzmiller R, Apelian R, Cho J, Ko D, Millet D. Asian women presenting with new onset refractory status epilepticus: cyclophosphamide-responsive NMDA receptor encephalitis without tumor. Epilepsy Curr. 2011;11.

255. Shrivastava M, Chouhan S, Navaid S. Plasma exchange as a therapeutic modality in a rare case of cryptogenic new onset refractory status epilepticus (NORSE). J Clin Diagn Res. 2017;11(7):ED33-ED4.

256. Soldatos A, Gorman M. Rituximab treatment in a girl with voltage-gated potassium channel associated FIRES (fever-induced refractory epileptic encephalopathy in school-aged children). J Neuroimmunol. 2012;253:13-4.

257. Thomas D, Livingston M, Currey K, Krumholz A. Anti-NMDA receptor encephalitis associated with an ovarian teratoma presenting as non convulsive status epilepticus with atypical ictal paroxysmal fast activity. Epilepsy Curr. 2012;1(Suppl. 1).

258. Ting IP, Abdul Halim S, Adnan A, Jaafar H. Status epilepticus as the initial presentation of antibody-negative Goodpasture's syndrome. BMJ Case Rep. 2017;2017: bcr2017219628.

259. Tripplet J, Vijayan S, MacDonald A, Lawn N, McLean-Tooke A, Bynevelt M, et al. Fulminant Anti-GAR antibody encephalitis, presenting with status epilepticus requiering aggressive immunosuppression. J Neuroimmunol. 2018;323:119-24.

260. van Baalen A, Häusler M, Plecko-Startinig B, Strautmanis J, Vlaho S, Gebhardt B, et al. Febrile infection-related epilepsy syndrome without detectable autoantibodies and response to immunotherapy: a case series and discussion of epileptogenesis in FIRES. Neuropediatrics. 2012;43:209-16.

261. Villani F, Spreafico R, Farina L, Giovagnoli A, Bernasconi P, Granata T. Positive response to immunomodulatory therapy in 
an adult patient with Rassmussen's encephalitis. Neurology. 2001;56(2):248-50.

262. Wilder-Smith E, Lim E, Tehoh H, Sharma V, Tan J, Chan B. The NORSE (new-onset refractory status epilepticus) syndrome: defining a disease entity. Ann Acad Med Singap. 2005;34(7):417-20.

263. Yamamoto D, Uchiyama T, Bunai T, Sato K, Shimizu T, Tanaka K, et al. Acute encephalitis with refractory partial status epilepticus treated with early immunotherapies including plasma exchange: a case report. Rinsho Shinkeigaku. 2014;54(9):715-20.

264. Yeo L, Loh P, Tan J, Chan Y. Different presentations and outcomes in NMDAR antibody encephalitis. Eur J Neurol. 2009;16:574.

265. Yoshida H, Furugori M, Kanatsuka Y, Yamaguchi S, Shigeta H. Case of anti-Hu antibody-mediated encephalopathy associated with ovarian cancer. J Obstet Gynaecol Res. 2019;45(9):1948-51.

\section{Affiliations}

\section{Laurent M. Willems $s^{1,2,3}$ (D) Sebastian Bauer ${ }^{1,2,3}$ D $\cdot$ Kolja Jahnke $^{2} \cdot$ Martin Voss $^{2,4,5}$ (D) $\cdot$ Felix Rosenow $^{1,2,3}$ (D) Adam Strzelczyk $\mathbf{k}^{1,2,3,6}$ iD}

1 Epilepsy Center Frankfurt Rhine-Main, Center of Neurology and Neurosurgery, Goethe-University Frankfurt, Schleusenweg 2-16, 60528 Frankfurt am Main, Germany

2 Department of Neurology, Goethe University Frankfurt, Frankfurt am Main, Germany

3 LOEWE Center for Personalized Translational Epilepsy Research (CePTER), Goethe University Frankfurt, Frankfurt am Main, Germany
4 Dr. Senckenberg Institute of Neuro-Oncology, Goethe University Frankfurt, University Hospital Frankfurt, Frankfurt am Main, Germany

5 Frankfurt Cancer Institute (FCI), Goethe University Frankfurt, Frankfurt am Main, Germany

6 Department of Neurology, Epilepsy Center Hessen, Philipps University Marburg, Marburg (Lahn), Germany 